\title{
A MODEL FOR CRACK GROWTH WITH BRANCHING AND KINKING
}

\author{
SIMONE RACCA \\ SISSA, VIA BONOMEA 265, 34136 TRIESTE, ITALY
}

\begin{abstract}
We study an evolution model for fractured elastic materials in the 2 -dimen-sional case, for which the crack path is not assumed to be known a priori. We introduce some general assumptions on the structure of the fracture sets suitable to remove the restrictions on the regularity of the crack sets and to allow for kinking and branching to develop. In addition we define the front of the fracture and its velocity. By means of a time-discretization approach, we prove the existence of a continuous-time evolution that satisfies an energy inequality and a stability criterion. The energy balance also takes into account the energy dissipated at the front of the fracture. The stability criterion is stated in the framework of Griffith's theory, in terms of the energy release rate, when the crack grows at least at one point of its front.
\end{abstract}

Keywords: quasistatic crack evolution, branching, kinking, Griffith's criterion.

2010 Mathematics Subject Classification: 35Q74, 49J40, 74A45, 74H20, 74R10.

\section{Contents}

1. Introduction 1

2. Notation 4

3. The admissible crack sets 5

4. The time-incremental problem 16

5. The continuous-time evolution 22

5.1. Velocity of the crack tips 27

6. Properties of the continuous-time evolution 33

6.1. Energy inequality 33

6.2. Energy release rate and Griffith's principle $\quad 37$

References 43

\section{INTRODUCTION}

Fracture mechanics represents at present an interesting and open field of mathematical investigation, although almost a century has passed since the seminal work by Griffith [22] on quasistatic brittle fracture evolution. During this lapse of time, many steps have been done towards a clearer understanding of the process and a

Preprint SISSA 45/2012/M (Revised version July 2013). 
more rigorous definition of the elements of the theory. Key steps in this improvement path deal with the restrictions which are part of the Griffith's model and with the concepts therein introduced.

Griffith's principle about evolution dwells on the idea that the crack growth is determined by the competition between the elastic energy of the unfractured body and the work needed to create a new crack or to extend an existing one. Its model is essentially 2-dimensional and requires the crack path to be known a priori.

In order to remove these restrictions, Francfort \& Marigo [21] propose a variational formulation which does not require the crack path to be preassigned and can be generalized to any dimension. The first existence result of a continuous-time evolution of such a model (without the path of the crack prescribed a priori) is given in [17] in the contest of antiplane linear elasticity, assuming the cracks to be finitely many compact connected sets of finite length. This result has then been extended to plane elasticity [10], to finite elasticity [15, 16], to a larger functional space [20].

Concerning Griffith's evolution principle, the main ingredient is represented by the energy release rate, i.e. the amount of elastic energy dissipated due to an infinitesimal increase of the crack. If $\Omega \subset \mathbb{R}^{2}$ is an elastic body, $\Gamma \subset \bar{\Omega}$ is the preassigned 1-dimensional crack path, and $\mathcal{E}(w, l)$ is the elastic energy related to a driving force $w$ acting on $\Omega$ and a connected fracture of length $l$ along $\Gamma$, then the energy release rate is formally defined as

$$
\mathcal{G}(w, l):=\lim _{h \rightarrow 0^{+}} \frac{\mathcal{E}(w, l)-\mathcal{E}(w, l+h)}{h} .
$$

The rigorous proof of existence of $\mathcal{G}$ is a non-trivial issue. The main technique makes use of the domain differentiation method: the evolving domain is mapped to a fixed one by means of smooth enough transformations. This approach requires the crack set to be sufficiently regular, in order to be able to construct the mentioned transformations. So far, the best result is obtained in [28], where existence of the energy release rate is proved for $C^{1,1}$ crack path and a quadratic elastic energy. In [23], the authors consider the case of nonlinear energies, with polyconvex energy densities.

In the 2-dimensional setting, in [25, 34, 27] models which also consider a dissipative term due to the rate of crack growth are presented. In [25, 34] the crack path is a sufficiently smooth preassigned curve, while in [27] the fracture set is only required to belong to a suitable class of compact 1-dimensional sets, containing a finite number of $C^{1,1}$ non-interacting curves. An interesting analysis of different models is described in [29].

Anyway, at present it is not clear how to deal with branching and kinking in a sufficiently general setting. Recently, Negri [30] showed the existence of $\mathcal{G}$ for piecewise $C^{1,1}$ cracks, i.e. in his setting the fracture is allowed to kink. His approach, by means of $\Gamma$-convergence, provides an integral representation of the energy release rate that depends on the kinking angle in an implicit way. Despite the importance of the result, unfortunately it is difficult to be used in order to choose among different criterion available for the description of the kinking phenomenon: maximum energy release rate, principle of local symmetry, ... A discussion on these topics is presented in $[11,12]$.

The main feature of the model we present here is that it addresses the issue about branching and kinking from a different perspective. We propose an evolution model 
for fractured elastic materials in the 2-dimensional antiplane case which allows those phenomena to take place and does not require the crack path to be known a priori. The idea is to introduce some general assumptions on the structure of the fracture sets which allow us to extend Griffith's theory to the case of branching and kinking and, at the same time, to define the front of the fracture and its velocity. The last two concepts are discussed in [26], where the authors introduce them by means of a variational approach and study models of crack growth in which the crack dissipation is a function of the front speed.

As a standard procedure, we consider first a discrete-time approximation $t \mapsto$ $\left(\Gamma_{\tau}(t), u_{\tau}(t)\right)$ driven by a boundary datum $w_{\tau}(t)$, where $\tau$ is the time-incremental step. Here $\Omega$ is the reference configuration of an elastic material, $\Gamma_{\tau}(t) \subset \bar{\Omega}$ represents the crack set, while $u_{\tau}(t)$ is the elastic displacement of the unfractured part $\bar{\Omega} \backslash \Gamma_{\tau}(t)$ of the material. At each incremental time-step, the minimum problem that selects the proper approximation takes into account terms related to the discrete velocity of the front. The discrete-time evolution of the system satisfies a first order stability condition in the framework of Griffith's theory, in terms of the energy release rate, and an energy balance inequality of the form

$$
\begin{aligned}
& \int_{\Omega \backslash \Gamma_{\tau}(b)}\left|\nabla u_{\tau}(b)\right|^{2} d x+\mathcal{H}^{1}\left(\Gamma_{\tau}(b)\right)+\int_{a}^{b} \sum_{x \in F_{\tau}(t)} v_{\tau}(x, t)^{2} d t \\
\leq & \int_{\Omega \backslash \Gamma_{\tau}(a)}\left|\nabla u_{\tau}(a)\right|^{2} d x+\mathcal{H}^{1}\left(\Gamma_{\tau}(a)\right)+\int_{a}^{b} \int_{\Omega \backslash \Gamma_{\tau}(t)} \nabla u_{\tau}(t) \cdot \nabla \dot{w}(t) d x d t+o(1),
\end{aligned}
$$

for two instants $0 \leq a<b \leq 1$, where $F_{\tau}(t)$ and $v_{\tau}(x, t)$ represent the front of the fracture at time $t$ and its discrete velocity at $x \in F_{\tau}(t)$, respectively. In the inequality above, the term

$$
\int_{a}^{b} \sum_{x \in F_{\tau}(t)} v_{\tau}(x, t)^{2} d t
$$

represents the energy dissipated at the crack front due to the speed of the crack growth. It also plays a role in the statement of the Griffith's principle.

We are able to recover a continuous-time rate-dependent evolution $(\Gamma(t), u(t))$ as limit of the $\left(\Gamma_{\tau}(t), u_{\tau}(t)\right)$ when the incremental step $\tau$ vanishes. An important ingredient is represented by the compactness of the family of admissible fracture sets, for which some geometrical constraints are necessary in order to control the germination of new branches of the crack and their reciprocal interaction during the process. The crack growth is irreversible, i.e. $\Gamma\left(t^{\prime}\right) \subset \Gamma(t)$ for any $t^{\prime}<t$. We also describe the notion of velocity of the crack front with two different approaches. One of them, obtained by means of an abstract setting, reminds that by Larsen, Ortiz \& Richardson in [26].

The continuous-time evolution satisfies an energy inequality analogous to (1.1). However, the main issue is the description of a stability criterion for the evolution. The presence of several branches of the crack requires a fine investigation of the approximation by discrete-time evolutions. We are able to recover a Griffith's principle as long as the front advances: if at an instant $t_{0}$ the velocity $v\left(p\left(t_{0}\right), t_{0}\right)$ of a point $p\left(t_{0}\right)$ of the front $F\left(t_{0}\right)$ is not null, then for a.e. $t<t_{0}$ and close to $t_{0}$ the following 
conditions are satisfied

$$
\begin{aligned}
& v(t, p(t)) \geq 0 \\
& \mathcal{G}(w(t), \Gamma(t), p(t)) \leq 1+v(t, p(t)) \\
& {[-\mathcal{G}(w(t), \Gamma(t), p(t))+1+v(t, p(t))] v(t, p(t))=0,}
\end{aligned}
$$

where $p(t)$ belongs to the front $F(t)$ of $\Gamma(t)$ and $p(t) \rightarrow p\left(t_{0}\right)$ as $t \nearrow t_{0}$. In (1.2), the function $\mathcal{G}(w, \Gamma, p)$ is the energy release rate relative to a time-dependent loading $w$, a crack $\Gamma$ and a tip $p$ of $\Gamma$.

When a branch of the crack does not grow, we cannot prove a stability condition like (1.2). The main difficulty dwells on the approximation procedure by the discretetime evolutions, as explained at the end of the paper.

As said, in the work we study the antiplane elasticity model. The results up to Subsection 6.1 can be treated in more general settings, like linearized and nonlinear elasticity, appealing to the stability results in [10,6] and [14] instead of Lemma 4.3 below. However, in Subsection 6.2 we will need an explicit formula for the energy release rate. In the antiplane case it is proved in [28], while it is lacking in the other regimes for our geometric setting (see [23, 24] in case of polyconvex elastic energy densities and preassigned crack path, and [11] for the linearized case).

We conclude this introduction with a final remark. To our knowledge, this is a first attempt to consider a model with branching and kinking, in the framework of Griffith's theory, and which considers the rate of growth of the crack. Nevertheless some work is still necessary in order to obtain a complete description of the growth process and to remove some mathematical constraints introduced for the well-posedness of the problem.

\section{NotATION}

Let $\Omega$ be a bounded connected open subset of $\mathbb{R}^{2}$, with Lipschitz boundary $\partial \Omega$. Let $\partial_{D} \Omega \subset \partial \Omega$ be relatively open, with $\mathcal{H}^{1}\left(\partial_{D} \Omega\right)>0$; we refer to $\partial_{D} \Omega$ as the Dirichlet part of the boundary. With $\mathcal{H}^{1}$ we mean the 1-dimensional Hausdorff measure.

We study an evolution problem in a fixed time interval $[0,1]$. On $\partial_{D} \Omega$ we prescribe a time-dependent boundary displacement which, at each instant $t \in[0,1]$, is given by the value of (the trace of) a function

$$
w \in H^{1}\left(0,1 ; H^{1}(\Omega)\right)
$$

at $t$.

The initial configuration is the couple $\left(u_{0}, \Gamma_{0}\right)$ where $u_{0} \in H^{1}\left(\Omega \backslash \Gamma_{0}\right)$ is solution to

$$
\begin{cases}\Delta u_{0}=0 & \text { in } \Omega \backslash \Gamma_{0} \\ \frac{\partial u_{0}}{\partial \mathbf{n}}=0 & \text { on } \Gamma_{0} \\ u_{0}=w(0) & \text { on } \partial_{D} \Omega\end{cases}
$$

and $\Gamma_{0}$ belongs to the class $\mathcal{S}$ of admissible cracks described below (see Definition 3.6). The class $\mathcal{S}$ is endowed with the topology induced by the Hausdorff metric defined on sets (see [5]). 
Considered a sequence of set $\Gamma_{k}$ that converges to a set $\Gamma$ in the Hausdorff topology as $k \rightarrow+\infty$, we will write

$$
\Gamma=\mathcal{H}_{-} \lim _{k \rightarrow+\infty} \Gamma_{k} \quad \text { or } \quad \Gamma_{k} \stackrel{\mathcal{H}}{\longrightarrow} \Gamma .
$$

By the notation $\langle\cdot, \cdot\rangle$ and $\|\cdot\|$ we understand the inner product and the norm in the Hilbert spaces $L^{2}(\Omega)$ or $L^{2}\left(\Omega ; \mathbb{R}^{2}\right)$.

Finally, the constant $C$ may vary also within the same proof and is independent of all the parameters, unless we explicitly write the dependence.

\section{The Admissible CRACK Sets}

In this section we introduce the geometrical setting of the model. We describe the class of admissible cracks and prove its compactness with respect to the Hausdorff convergence. Throughout the section, we comment on the meaning of some mathematical and geometrical constraints necessary to obtain this result.

We consider a slight modification of the family of curves $\mathcal{R}_{\eta}$ introduced in [28, 27].

Definition 3.1. For any $\eta>0, \mathcal{R}_{\eta}$ denotes the set of compact curves $\Gamma$ of class $C^{1,1}$ in $\bar{\Omega}$ such that

(i) $\mathcal{H}^{1}(\Gamma)>0$ and $\Gamma \subset \subset \Omega$;

(ii) for every point $x \in \Gamma$ there exist two open balls $B_{1}, B_{2}$ of radius $\eta$, such that $\left(B_{1} \cup B_{2}\right) \cap(\Gamma \cup \partial \Omega)=\varnothing$ and $\bar{B}_{1} \cap \bar{B}_{2}=\{x\}$.

Proposition 3.2. The following facts hold:

(i) every curve $\Gamma \in \mathcal{R}_{\eta}$ is simple.

(ii) Fix $\Gamma_{0} \in \mathcal{R}_{\eta}$. Then the set

$$
\left\{\Gamma \in \mathcal{R}_{\eta}: \Gamma_{0} \subset \Gamma\right\}
$$

is compact with respect to the Hausdorff convergence.

(iii) Consider $\Gamma \in \mathcal{R}_{\eta}$ and its arc-length parametrization $\gamma$. Then, set $L_{\Gamma}=$ $\mathcal{H}^{1}(\Gamma)$, it is $\gamma \in W^{2, \infty}\left(\left(0, L_{\Gamma}\right) ; \mathbb{R}^{2}\right)$.

(iv) There exists a constant $C>0$ such that

$$
\|\gamma\|_{W^{2, \infty}\left(\left(0, L_{\Gamma}\right) ; \mathbb{R}^{2}\right)} \leq C
$$

for any $\Gamma \in \mathcal{R}_{\eta}$.

(v) Let $\left(\Gamma_{k}\right)_{k} \subset \mathcal{R}_{\eta}$ be such that $\Gamma_{k} \stackrel{\mathcal{H}}{\longrightarrow} \Gamma$. Then $\mathcal{H}^{1}\left(\Gamma_{k}\right) \rightarrow \mathcal{H}^{1}(\Gamma)$.

(vi) Let $\left(\Gamma_{k}\right)_{k} \subset \mathcal{R}_{\eta}$ be such that $\Gamma_{k} \stackrel{\mathcal{H}}{\rightarrow} \Gamma$, with $L=\mathcal{H}^{1}(\Gamma)>0$. Let $L_{k}=\mathcal{H}^{1}\left(\Gamma_{k}\right)$, and $\gamma_{k}, \gamma$ be the arc-length parametrizations of $\Gamma_{k}$ and $\Gamma$, respectively. Define $\tilde{\gamma}_{k}(s):=\gamma_{k}\left(\frac{L_{k}}{L} s\right)$. Then $\tilde{\gamma}_{k} \in W^{2, \infty}\left((0, L) ; \mathbb{R}^{2}\right)$ and

$$
\tilde{\gamma}_{k} \rightarrow \gamma
$$

weakly in $W^{2, \infty}\left((0, L) ; \mathbb{R}^{2}\right)$. In particular, by the fundamental theorem of calculus, it holds that

$$
\begin{array}{ll}
\tilde{\gamma}_{k} \rightarrow \gamma & \text { pointwise } \\
\dot{\tilde{\gamma}}_{k} \rightarrow \dot{\gamma} & \text { pointwise. }
\end{array}
$$


Proof. Properties 3.2.(i), 3.2.(ii), 3.2.(iii), 3.2.(iv) and 3.2.(vi) are already proved in [28].

Concerning 3.2.(v), if $\mathcal{H}^{1}(\Gamma)>0$, then the conclusion follows by applying 3.2.(iv) and (3.2) in the formula for the length of a $C^{1}$ curve.

If $\mathcal{H}^{1}(\Gamma)=0$, then $\Gamma=\{x\}$ since it is connected. For any $r>0$, it is $\Gamma_{k} \subset B_{r}(x)$ for $k$ sufficiently large. Then the bound on the curvature for $\Gamma_{k} \in \mathcal{R}_{\eta}$ implies that $\mathcal{H}^{1}\left(\Gamma_{k}\right) \leq C r$ for a constant $C$ independent of $r$, so that $\mathcal{H}^{1}\left(\Gamma_{k}\right) \rightarrow 0$ as $k \rightarrow+\infty$.

In the following, we call endpoints of $\Gamma \in \mathcal{R}_{\eta}$ the points $\gamma(0)$ and $\gamma(L)$, where $\gamma$ is the arc-length parametrization of $\Gamma$ and $L=\mathcal{H}^{1}(\Gamma)$.

The admissible crack sets will be finite unions of elements in $\mathcal{R}_{\eta}$, satisfying some topological restrictions in order to control the phenomena of branching and kinking. To define a proper class of cracks, for any curve in $\mathcal{R}_{\eta}$ we introduce two kinds of neighbourhoods, called 1-sided pencil-like neighbourhood and 2-sided pencil-like neighbourhood, which depend on two parameters which will not change along the paper:

$$
\beta \in(0, \eta) \text { and } \theta \in(0, \pi / 2) .
$$

Fixed $\Gamma \in \mathcal{R}_{\eta}$, set $L:=\mathcal{H}^{1}(\Gamma)$ and $\gamma:[0, L] \rightarrow \mathbb{R}^{2}$ the arc-length parametrization of $\Gamma$. Consider the rectangle

$$
R=(0, L) \times(-\beta, \beta)
$$

and the extended one

$$
R_{e}=(0, L+\beta) \times(-\beta, \beta) .
$$

Define the maps $\Phi: R \rightarrow \mathbb{R}^{2}$ and $\Phi_{e}: R_{e} \rightarrow \mathbb{R}^{2}$ as

$$
\Phi(s, z):=\gamma(s)+z \dot{\gamma}(s)^{\perp} \quad \text { if }(s, z) \in R
$$

and

$\Phi_{e}(s, z):= \begin{cases}\Phi_{e}(s, z):=\gamma(s)+z \dot{\gamma}(s)^{\perp} & \text { if }(s, z) \in R \\ \Phi(s, z):=\gamma(L)+(s-L) \dot{\gamma}(L)+z \dot{\gamma}(L)^{\perp} & \text { if }(s, z) \in[L, L+\beta) \times(-\beta, \beta) .\end{cases}$

By the regularity of $\gamma$ (see Proposition 3.2), the maps $\Phi$ and $\Phi_{e}$ are homeomorphisms from $R$ into $\Phi(R)$ and from $R_{e}$ into $\Phi_{e}\left(R_{e}\right)$, respectively.

We consider the subset of $R$

$$
P_{2}:=\{(s, z) \in R: 0<s<L,|z|<\min \{s \tan \theta, \beta,(L-s) \tan \theta\}\}
$$

and define the 2-sided pencil-like neighbourhood of $\Gamma$ (see Fig. 1) as

$$
\mathcal{P}_{2}(\Gamma):=\Phi\left(P_{2}\right) \text {. }
$$

In coordinates, it is

$$
\mathcal{P}_{2}(\Gamma)=\left\{\gamma(s)+z \dot{\gamma}(s)^{\perp}: 0<s<L,|z|<\min \{s \tan \theta, \beta,(L-s) \tan \theta\}\right\} .
$$

We consider the subset of $R_{e}$

$$
\begin{aligned}
P_{1}:= & \{(s, z) \in R: 0<s \leq L,|z|<\min \{s \tan \theta, \beta\}\} \\
& \cup\left\{(s, z) \in R_{e}: L \leq s<L+\beta,|z|<\min \left\{s \tan \theta, \sqrt{\beta^{2}-(s-L)^{2}}\right\}\right\}
\end{aligned}
$$

and define the 1-sided pencil-like neighbourhood of $\Gamma$ (see Fig. 2) as

$$
\mathcal{P}_{1}(\Gamma, p):=\Phi_{e}\left(P_{1}\right)
$$



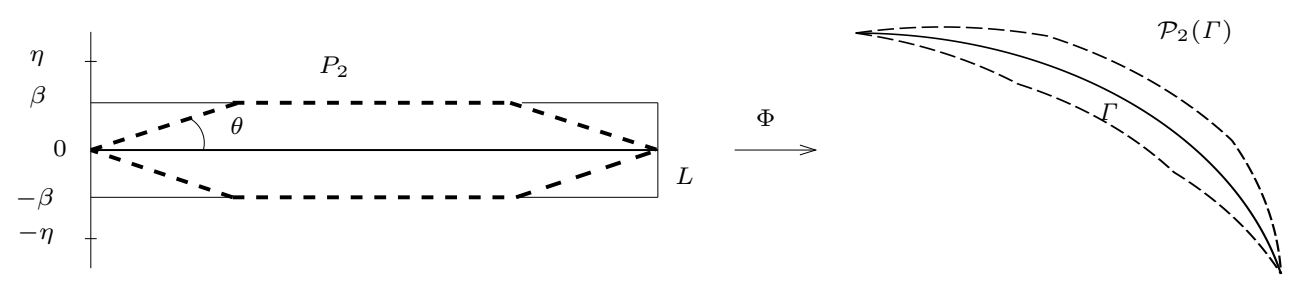

Figure 1: The 2-sided pencil-like neighbourhood $\mathcal{P}_{2}(\Gamma)$ for a curve $\Gamma \in \mathcal{R}_{\eta}$.

with $p=\gamma(0)$. In coordinates, it is

$$
\begin{gathered}
\mathcal{P}_{1}(\Gamma, p)=\left\{\gamma(s)+z \dot{\gamma}(s)^{\perp}: 0<s \leq L,|z|<\min \{s \tan \theta, \beta\}\right\} \\
\cup\left\{\gamma(L)+(s-L) \dot{\gamma}(L)+z \dot{\gamma}(L)^{\perp}: L \leq s<L+\beta\right. \\
\text { and } \left.|z|<\min \left\{s \tan \theta, \sqrt{\beta^{2}-(s-L)^{2}}\right\}\right\} .
\end{gathered}
$$
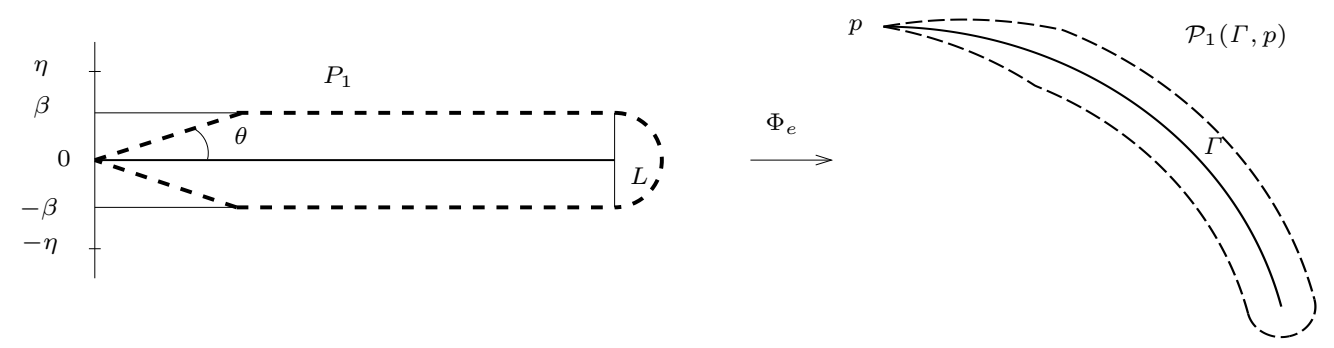

Figure 2: The 1-sided pencil-like neighbourhood $\mathcal{P}_{1}(\Gamma, p)$ for a curve $\Gamma \in \mathcal{R}_{\eta}$.

Remark 3.3. With abuse of terminology, we use the name neighbourhood for the open sets $\mathcal{P}_{1}$ and $\mathcal{P}_{2}$. They are not neighbourhoods of $\Gamma$, but only of $\Gamma \backslash\left\{p_{0}, p_{1}\right\}$ in case of $\mathcal{P}_{2}(\Gamma)$ and of $\Gamma \backslash\left\{p_{0}\right\}$ in case of $\mathcal{P}_{1}\left(\Gamma, p_{0}\right)$, where $p_{0}, p_{1}$ are the endpoints of $\Gamma$. In particular, the following holds: if $\mathcal{P}_{2}\left(\Gamma_{1}\right) \cap \Gamma_{2}=\varnothing$ and $\Gamma_{1} \cap \Gamma_{2} \neq \varnothing$, then they intersect in (one of or both) their endpoints. Similarly, if $\mathcal{P}_{1}\left(\Gamma_{1}, p_{0}\right) \cap \Gamma_{2}=\varnothing$ and $\Gamma_{1} \cap \Gamma_{2} \neq \varnothing$, then they intersect in $p_{0}$.

We consider finite unions of curves in $\mathcal{R}_{\eta}$ satisfying the following properties:

$$
K=\bigcup_{j=1}^{m} \widetilde{K}_{j}
$$

such that

(i) $K$ is connected; 
(ii) $\widetilde{K}_{j} \in \mathcal{R}_{\eta}$ for any $j$;

(iii) if $\widetilde{K}_{j} \cap \widetilde{K}_{l} \neq \varnothing$ for $j \neq l$, then they intersect in (one of or both) their endpoints;

(iv) if $\widetilde{K}_{i} \cup \widetilde{K}_{j} \in \mathcal{R}_{\eta}$, then there exists $\widetilde{K}_{l}, l \neq i, j$, such that $\widetilde{K}_{i} \cap \widetilde{K}_{j} \cap \widetilde{K}_{l} \neq \varnothing$.

(v) let $p_{0}, p_{1}$ be the endpoints of $\widetilde{K}_{j}$. Assume that $p_{0} \in \widetilde{K}_{j} \cap \widetilde{K}_{l_{0}}$ and $p_{1} \in$ $\widetilde{K}_{j} \cap \widetilde{K}_{l_{1}}$ for some $l_{0}, l_{1} \neq j$. Then

$$
\mathcal{P}_{2}\left(\widetilde{K}_{j}\right) \cap \widetilde{K}_{l}=\varnothing
$$

for every $l \neq j$;

(vi) let $p_{0}, p_{1}$ be the endpoints of $\widetilde{K}_{j}$. Assume that $p_{0} \in \widetilde{K}_{j} \cap \widetilde{K}_{l_{0}}$ for some $l_{0} \neq j$ and $p_{1} \notin \widetilde{K}_{l}$ for any $l \neq j$. Then

$$
\mathcal{P}_{1}\left(\widetilde{K}_{j}, p_{0}\right) \cap \widetilde{K}_{l}=\varnothing
$$

for every $l \neq j$.

Each $\widetilde{K}^{j}$ is called a branch of $K$.

Definition 3.4. We divide the points of a set $K$ as in (3.3) in three groups:

- the set $T_{K}$ of crack tip points: $x \in K$ belongs to $T_{K}$ if there exists $r>0$ such that $K \cap \overline{B_{r}(x)}$ is an element of $\mathcal{R}_{\eta}$ with endpoint $x$.

- The set $S_{K}$ of singular points: $x \in K$ belongs to $S_{K}$ if there exist two unit vectors $v_{1}, v_{2} \in \mathbb{R}^{2}$ tangent to $K$ at $x$ such that $v_{1} \cdot v_{2} \neq \pm 1$.

- The set $R_{K}$ of regular points: $x \in K$ belongs to $R_{K}$ if there exists $r>0$ such that $K \cap \overline{B_{r}(x)}$ is an element of $\mathcal{R}_{\eta}$ and $x$ in the relative interior of $K$.

Remark 3.5. Conditions (3.3).(iii) and (3.3).(iv) represent a sort of "maximality" condition of each branch in the class $\mathcal{R}_{\eta}$ with respect to inclusion.

Conditions (3.3).(v) and (3.3).(vi) are mathematical restrictions, which will be necessary to prove compactness of a suitable class of crack sets. We require that each branch is surrounded by an off-limit zone for other branches; it is represented by the pencil-like neighbourhoods. Around one of (both) the tips, the 1-sided (2-sided) pencil-like neighbourhoods have the shape of a curvilinear triangle with vertex at the tip and vertex angle of $2 \theta$. The triangular shape is necessary in order to permit the branching phenomenon at the tip; the condition on the angle bounds the number of branches that can develop from each singular point, as proved in Lemma 3.9 below.

For any $K$ of this form we can define the sets $I_{1}(K)$ and $I_{2}(K)$ such that

$$
\begin{array}{ll}
\widetilde{K}_{j} \in I_{1}(K) & \text { if and only if } \widetilde{K}_{j} \text { satisfies the assumption in (3.3).(vi) } \\
\widetilde{K}_{l} \in I_{2}(K) & \text { if and only if } \widetilde{K}_{l} \text { satisfies the assumption in (3.3).(v). }
\end{array}
$$

It is:

- $T_{\Gamma}$ is the set of endpoints $p_{1}$ of $\widetilde{K}_{j}$, for some $j \in I_{1}\left(K_{l}\right)$;

- $S_{\Gamma}$ is the set of endpoints $p_{0}, p_{1}$ of $\widetilde{K}_{j}$, for some $j \in I_{2}\left(K_{l}\right)$;

- $R_{\Gamma}$ is the set $\Gamma \backslash\left(T_{\Gamma} \cup S_{\Gamma}\right)$.

Definition 3.6. Let $\delta, \lambda$ be positive constants, with

$$
\delta \geq \beta\left(\frac{2}{\tan \theta}+1\right) \text { and } \lambda \geq \frac{\beta}{\tan \theta} .
$$


We define the class $\mathcal{S}$ of admissible cracks as the set of curves $\Gamma$ of the form

$$
\Gamma=\bigcup_{j=1}^{N} K_{j}
$$

such that $N \in \mathbb{N}$ and

(i) each $K_{j}$ is of the form (3.3) and verifies Conditions (3.3).(i)-(3.3).(vi);

(ii) it is

$$
\begin{array}{lll}
K_{j} \cap \mathcal{P}_{1}\left(\widetilde{K}_{l}, p_{0}\right)=\varnothing & \text { for every } \widetilde{K}_{l} \in I_{1}\left(K_{m}\right) \text { and } m \neq j \\
K_{j} \cap \mathcal{P}_{2}\left(\widetilde{K}_{l}\right)=\varnothing & \text { for every } \widetilde{K}_{l} \in I_{2}\left(K_{m}\right) \text { and } m \neq j ;
\end{array}
$$

(iii) $\mathcal{H}^{1}\left(K_{j}\right) \geq \lambda$ for every $j$;

(iv) defined $S_{\Gamma}:=\bigcup_{j=1}^{N} S_{K_{j}}$, for every $x_{1}, x_{2} \in S_{\Gamma}$ with $x_{1} \neq x_{2}$ it is

$$
\left|x_{1}-x_{2}\right| \geq \delta \text {. }
$$

An example of an element $\Gamma \in \mathcal{S}$ is showed in Fig. 3.

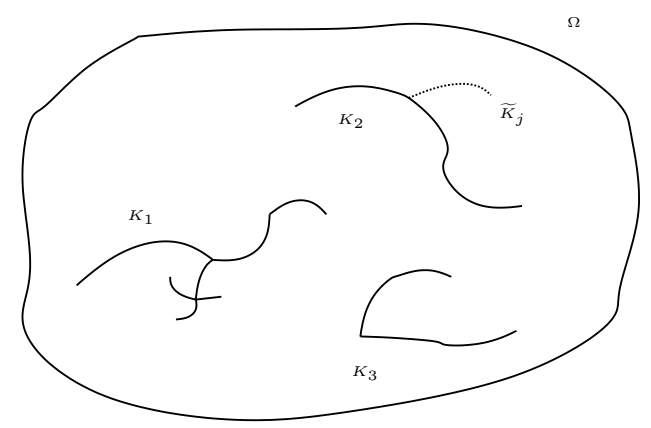

Figure 3: A domain $\Omega \subset \mathbb{R}^{2}$ with crack set $\Gamma \in \mathcal{S}$ of the form $\Gamma=K_{1} \cup K_{2} \cup K_{3}$. The dotted curve $\tilde{K}_{j}$ is a branch of $K_{2}$.

Remark 3.7. Let us comment immediately on the assumptions (3.5). Notice that, if $\widetilde{K} \in \mathcal{R}_{\eta}, \gamma$ is its arc-length parametrization and $\mathcal{H}^{1}(\widetilde{K}) \geq \beta(1+1 / \tan \theta)$, then for every $s \geq \beta(1+1 / \tan \theta)$ it is

$$
B_{\beta}(\gamma(s)) \subset \mathcal{P}_{1}(\widetilde{K}, \gamma(0)) .
$$

If $\mathcal{H}^{1}(\widetilde{K}) \geq \delta$, then there exists $x \in \partial \mathcal{P}_{2}(\widetilde{K})$ with $\operatorname{dist}(x, \widetilde{K})=\beta$. Indeed, this is true for

$$
x=\gamma(s)+z \dot{\gamma}(s)^{\perp}
$$

with $\beta / \tan \theta \leq s \leq \beta / \tan \theta+\beta$, for which $z= \pm \beta$.

The constraint (3.5) on $\lambda$ and Condition 3.6.(iii) assure that, if a connected component $K$ contains only one branch, i.e. $K \in \mathcal{R}_{\eta}$, then at each tip of $K$ the 1 -sided pencil-like neighbourhood contains a half-ball of radius $\beta$.

The main result of the section is the following theorem. Its proof is achieved after several lemmas, which will clarify the geometric meaning of the parameters and of the objects introduced for the definition of the class $\mathcal{S}$. 
Theorem 3.8. The class $\mathcal{S}$ is compact with respect to the Hausdorff convergence.

Lemma 3.9. There exists a constant $M \in \mathbb{N}$ such that every $\Gamma \in \mathcal{S}$ has at most $M$ branches. In addition, also the number of singular points and tip points is uniformly bounded.

Proof. By (3.7), in $\bar{\Omega}$ there can be at most

$$
\frac{1}{\frac{\sqrt{3}}{4} \delta^{2}}|\Omega|
$$

singular points, where $\sqrt{3} \delta^{2} / 4$ is the area of an equilateral triangle of side $\delta$.

By Conditions (3.3).(v) and (3.3).(vi) on the 1-sided and 2-sided pencil-like neighbourhoods, from each singular point at most $2 \pi / \theta$ branches can develop.

Therefore, $\frac{2 \pi}{\theta} \frac{\sqrt{3}}{4 \delta^{2}}|\Omega|$ is the maximum number of branches for an element $\Gamma \in \mathcal{S}$.

The final statement is an easy consequence of the previous part: the number of singular points and tip points is bounded by the number of equilateral triangles of side $\delta$ and by the number of branches, respectively.

Lemma 3.10. There exists a positive constant $C$ such that $\mathcal{H}^{1}(\Gamma) \leq C$ for every $\Gamma \in \mathcal{S}$.

Proof. Apply Proposition 3.2.(iv) and Lemma 3.9.

Lemma 3.11. There exists an increasing function $\rho:(0,+\infty) \rightarrow(0, \beta]$ satisfying the following property: if $K \in \mathcal{R}_{\eta}$ and $\gamma$ is its arc-length parametrization, then

$$
B_{\rho(s)}(\gamma(s)) \subset \mathcal{P}_{1}(K, \gamma(0)),
$$

for every $s \in\left(0, \mathcal{H}^{1}(K)\right]$.

Proof. By elementary geometrical arguments, it is not difficult to observe that the bound on the curvature in the class $\mathcal{R}_{\eta}$ implies that

$$
\rho(s)=\frac{\tan \theta}{2} s
$$

satisfies the above property.

Lemma 3.12. Let $\left(\Gamma_{k}\right)_{k} \subset \mathcal{R}_{\eta}$ be such that $\Gamma_{k} \stackrel{\mathcal{H}}{\longrightarrow} \Gamma$ and $\mathcal{H}^{1}(\Gamma)>0$. Then

$$
\partial \mathcal{P}_{2}\left(\Gamma_{k}\right) \stackrel{\mathcal{H}}{\longrightarrow} \partial \mathcal{P}_{2}(\Gamma) \quad \text { and } \quad \overline{\mathcal{P}_{2}\left(\Gamma_{k}\right)} \stackrel{\mathcal{H}}{\longrightarrow} \overline{\mathcal{P}_{2}(\Gamma)} .
$$

If $p_{k}^{0} \rightarrow p^{0}$, then

$$
\partial \mathcal{P}_{1}\left(\Gamma_{k}, p_{k}^{0}\right) \stackrel{\mathcal{H}}{\longrightarrow} \partial \mathcal{P}_{1}\left(\Gamma, p^{0}\right) \quad \text { and } \quad \overline{\mathcal{P}_{1}\left(\Gamma_{k}, p_{k}^{0}\right)} \stackrel{\mathcal{H}}{\longrightarrow} \overline{\mathcal{P}_{1}\left(\Gamma, p^{0}\right)} .
$$

Proof. By the compactness property for $\mathcal{R}_{\eta}, \Gamma$ belongs to $\mathcal{R}_{\eta}$. We now adopt the notation of Proposition 3.2.

We consider first the case of $\partial \mathcal{P}_{2}$. In terms of the parametrization $\tilde{\gamma}_{k}$, it is

$\mathcal{P}_{2}\left(\Gamma_{k}\right)=\left\{\tilde{\gamma}_{k}(s)+\frac{L}{L_{k}} z \dot{\tilde{\gamma}}_{k}(s)^{\perp}: 0<s<L,|z|<\min \left\{\frac{L_{k}}{L} s \tan \theta, \beta, \frac{L_{k}}{L}(L-s) \tan \theta\right\}\right\}$

To obtain the claim, we verify the Kuratowski convergence, which in our setting is equivalent to the Hausdorff convergence (see for example [5]). 
- First we show that, given a sequence $y_{j} \in \partial \mathcal{P}_{2}\left(\Gamma_{k_{j}}\right)$ with $y_{j} \rightarrow y$ as $j \rightarrow+\infty$, then $y \in \partial \mathcal{P}_{2}(\Gamma)$. By definition of $\mathcal{P}_{2}$,

$$
y_{j}=\tilde{\gamma}_{k_{j}}\left(s_{j}\right)+\frac{L}{L_{k_{j}}} z_{j} \dot{\tilde{\gamma}}_{k_{j}}\left(s_{j}\right) .
$$

Up to subsequences, $s_{j} \rightarrow s \in[0, L]$. By the bound $\left\|\tilde{\gamma}_{k_{j}}\right\|_{W^{2, \infty}\left((0, L) ; \mathbb{R}^{2}\right)} \leq C,(3.1)$, and (3.2), we have

$$
\tilde{\gamma}_{k_{j}}\left(s_{j}\right) \rightarrow \gamma(s) \quad \text { and } \quad \dot{\tilde{\gamma}}_{k_{j}}\left(s_{j}\right) \rightarrow \dot{\gamma}(s) .
$$

In order to conclude, we should consider different scenarios:

$$
\begin{gathered}
\beta<\min \{s \tan \theta,(L-s) \tan \theta\} \\
s \tan \theta \leq \beta \leq(L-s) \tan \theta \\
(L-s) \tan \theta \leq \beta \leq s \tan \theta .
\end{gathered}
$$

We only consider the first case, for the others it is enough to argue similarly. Since $L_{k_{j}} / L \rightarrow 1$ and $s_{j} \rightarrow s$, it is $\beta<\min \left\{\frac{L_{k_{j}}}{L} s_{j} \tan \theta, \frac{L_{k_{j}}}{L}\left(L-s_{j}\right) \tan \theta\right\}$ for $j$ sufficiently large, so that $z_{j}=\beta$. Then, by (3.8), we obtain that $y=\gamma(s)+\beta \dot{\gamma}(s)$, which belongs to $\partial \mathcal{P}_{2}(\Gamma)$.

- Given $y=\gamma(s)+z \dot{\gamma}(s) \in \partial \mathcal{P}_{2}(\Gamma)$, we have to construct a sequence $y_{k} \in \partial \mathcal{P}_{2}\left(\Gamma_{k}\right)$ converging to $y$. Define

$$
y_{k}:=\tilde{\gamma}_{k}(s)+\frac{L}{L_{k}} z_{k} \dot{\tilde{\gamma}}_{k}(s) \in \partial \mathcal{P}_{2}\left(\Gamma_{k}\right)
$$

where, for $k$ large we set

$*$ if $\beta<\min \left\{\frac{L_{k}}{L} s \tan \theta, \frac{L_{k}}{L}(L-s) \tan \theta\right\}$, then $z_{k}=\beta$;

$*$ if $\frac{L_{k}}{L} s \tan \theta \leq \beta \leq \frac{L_{k}}{L}(L-s) \tan \theta$, then $z_{k}=\min \left\{\beta, \frac{L_{k}}{L} s \tan \theta\right\}$;

$*$ if $\frac{L_{k}}{L}(L-s) \tan \theta \leq \beta \leq \frac{L_{k}}{L} s \tan \theta$, then $z_{k}=\min \left\{\beta, \frac{L_{k}}{L}(L-s) \tan \theta\right\}$.

Using the pointwise convergence of $\tilde{\gamma}_{k}$ and $\dot{\tilde{\gamma}}_{k}$, it is easy to verify that $y_{k} \rightarrow y$.

For the case of $\partial \mathcal{P}_{1}$, in terms of $\tilde{\gamma}_{k}$, with $\tilde{\gamma}_{k}(0)=p_{k}^{0}$, the set $\mathcal{P}_{1}\left(\Gamma_{k}, p_{k}^{0}\right)$ is

$$
\begin{aligned}
\mathcal{P}_{1}\left(\Gamma_{k}, p_{k}\right)= & \left\{\tilde{\gamma}_{k}(s)+\frac{L}{L_{k}} z \dot{\tilde{\gamma}}_{k}(s)^{\perp}: 0<s<L,|z|<\min \left\{\frac{L_{k}}{L} s \tan \theta, \beta\right\}\right\} \\
& \cup\left\{\tilde{\gamma}_{k}(L)+(s-L) \dot{\tilde{\gamma}}_{k}(L)+\frac{L}{L_{k}} z \dot{\tilde{\gamma}}_{k}(L)^{\perp}: L<s<L+\frac{L}{L_{k}} \beta\right. \\
& \text { and } \left.|z|<\min \left\{\frac{L_{k}}{L} s \tan \theta, \sqrt{\beta^{2}-\frac{L_{k}^{2}}{L^{2}}(s-L)^{2}}\right\}\right\} .
\end{aligned}
$$

The proof follows the steps of the previous one, with the following differences.

- Let $y_{j} \in \partial \mathcal{P}_{1}\left(\Gamma, p_{k_{j}}^{0}\right)$ be such that $y_{j} \rightarrow y$. It is $y_{j}=\gamma_{k_{j}}\left(s_{j}\right)+x_{j}$ and, up to subsequences, $s_{j} \rightarrow s$, where

$*$ if $0 \leq s<L$, then for $j$ large

$$
x_{j}=\frac{L}{L_{k_{j}}} z_{j} \dot{\tilde{\gamma}}_{k_{j}}\left(s_{j}\right)^{\perp}
$$


$*$ if $L<s \leq L+\beta$, then for $j$ large

$$
x_{j}=\left(s_{j}-L\right) \dot{\tilde{\gamma}}_{k_{j}}(L)+\frac{L}{L_{k_{j}}} z_{j} \dot{\tilde{\gamma}}_{k_{j}}(L)^{\perp} ;
$$

* if $s=L$, then there exists a further subsequence such that either (3.9) or (3.10) hold for every term of the subsequence.

- Given $y \in \partial \mathcal{P}_{1}\left(\Gamma, p^{0}\right)$, we write $y=\gamma(s)+x$, with

$$
\begin{array}{ll}
x=z \dot{\gamma}(s)^{\perp} & \text { if } 0 \leq s \leq L \\
x=(s-L) \dot{\gamma}(L)+z \dot{\gamma}(L)^{\perp} & \text { if } L \leq s \leq L+\beta .
\end{array}
$$

Then one defines $y_{k} \in \partial \mathcal{P}_{1}\left(\Gamma_{k}, p_{k}^{0}\right)$ by considering $\tilde{\gamma}_{k}(s), \dot{\tilde{\gamma}}_{k}(s)$ and arguing similarly as for $\mathcal{P}_{2}$ in order to choose $z_{k}$ appropriately.

Similar arguments hold for the closed sets $\overline{\mathcal{P}_{2}\left(\Gamma_{k}\right)}$ and $\overline{\mathcal{P}_{1}\left(\Gamma_{k}, p_{k}^{0}\right)}$.

Lemma 3.13. Let $\left(\Gamma_{k}^{1}\right)_{k},\left(\Gamma_{k}^{2}\right)_{k} \subset \mathcal{R}_{\eta}$ be two sequences such that

$$
\Gamma_{k}^{1} \stackrel{\mathcal{H}}{\longrightarrow} \Gamma^{1} \quad \text { and } \quad \Gamma_{k}^{2} \stackrel{\mathcal{H}}{\longrightarrow} \Gamma^{2}
$$

and assume that $\mathcal{H}^{1}\left(\Gamma^{1}\right)>0$. The following properties hold:

- if for every $k$

$$
\begin{aligned}
& \mathcal{P}_{1}\left(\Gamma_{k}^{1}, p_{k}\right) \cap \Gamma_{k}^{2}=\varnothing \\
& p_{k} \rightarrow p \quad \text { with } \quad p_{k} \in T_{\Gamma_{k}^{1}},
\end{aligned}
$$

then $p \in T_{\Gamma^{1}}$ and $\mathcal{P}_{1}\left(\Gamma^{1}, p\right) \cap \Gamma^{2}=\varnothing$;

- if $\mathcal{P}_{2}\left(\Gamma_{k}^{1}\right) \cap \Gamma_{k}^{2}=\varnothing$ for every $k$, then $\mathcal{P}_{2}\left(\Gamma^{1}\right) \cap \Gamma^{2}=\varnothing$.

Proof. Being $p_{k} \in T_{\Gamma_{k}^{1}}$, it is $p_{k}=\tilde{\gamma}_{k}(0)$ for the arc-length parametrization $\gamma_{k}$. Since $\tilde{\gamma}_{k}(0) \rightarrow \gamma(0)$ by Proposition 3.2, it is $p \in T_{\Gamma^{1}}$.

By contradiction, assume that there exists $x \in \mathcal{P}_{1}\left(\Gamma^{1}, p^{1}\right) \cap \Gamma^{2}$. Let $r>0$ be such that $\operatorname{dist}\left(x, \partial \mathcal{P}_{1}\left(\Gamma^{1}, p^{1}\right)\right)=4 r$. By (3.11), there exists a sequence $x_{k} \in \Gamma_{k}^{2}$ converging to $x$; in particular $x_{k} \in B_{r}(x)$ for $k$ large. By Lemma 3.12, for $k$ large enough we have $B_{r}(x) \subset \mathcal{P}_{1}\left(\Gamma_{k}^{1}, p_{k}^{1}\right)$, so that

$$
x_{k} \in \Gamma_{k}^{2} \cap B_{r}(x) \subset \Gamma_{k}^{2} \cap \mathcal{P}_{1}\left(\Gamma_{k}^{1}, p_{k}^{1}\right),
$$

in contradiction to (3.12).

The second property can be proved in a similar manner.

Lemma 3.14. Let $\Gamma_{k} \in \mathcal{S}$ be a sequence converging to $\Gamma$ in the Hausdorff metric. Then $\mathcal{H}^{1}\left(\Gamma_{k}\right) \rightarrow \mathcal{H}^{1}(\Gamma)$.

Proof. Without loss of generality, we can assume that

$$
\Gamma_{k}=\bigcup_{i=1}^{N} \widetilde{K}_{k}^{i}
$$

with $\widetilde{K}_{k}^{i}$ branches of $\Gamma_{k}$ and

$$
\widetilde{K}_{k}^{i} \stackrel{\mathcal{H}}{\longrightarrow} \hat{K}^{i}
$$

for some $N \leq M$ ( $M$ given in Lemma 3.9). Indeed, if this is not the case, for every $N \leq M$ we consider the subsequence $\Gamma_{N, k}$ of elements having $N$ branches and, up to relabeling, we can assume that (3.14) holds. 
Firstly notice that, having $\Gamma_{k}$ a uniformly bounded number of connected components, by Gołab's Theorem [5, Theorem 4.4.17] we obtain immediately

$$
\mathcal{H}^{1}(\Gamma) \leq \liminf _{k \rightarrow+\infty} \mathcal{H}^{1}\left(\Gamma_{k}\right) .
$$

If $\mathcal{H}^{1}\left(\hat{K}^{i}\right)=0$, then $\hat{K}^{i}=\left\{x^{i}\right\}$. We argue as in the proof of 3.2.(v) of Proposition 3.2: the bound on the curvature for $\widetilde{K}_{k}^{i} \in \mathcal{R}_{\eta}$ and (3.14) imply that $\mathcal{H}^{1}\left(\widetilde{K}_{k}^{i}\right) \rightarrow$ 0 .

If for $i \neq l$ it is $\mathcal{H}^{1}\left(\hat{K}^{i}\right), \mathcal{H}^{1}\left(\hat{K}^{l}\right)>0$, then Lemma 3.13 and Remark 3.3 imply that

$$
\hat{K}^{i} \cap \hat{K}^{l} \subset\left\{p_{0}^{i}, p_{1}^{i}\right\}
$$

with $p_{0}^{i}, p_{1}^{i}$ endpoints of $\hat{K}^{i}$. Hence $\mathcal{H}^{1}\left(\hat{K}^{i} \cup \hat{K}^{l}\right)=\mathcal{H}^{1}\left(\hat{K}^{i}\right)+\mathcal{H}^{1}\left(\hat{K}^{l}\right)$. Applying Proposition 3.2.(v),

$$
\mathcal{H}^{1}\left(\hat{K}^{i}\right)=\lim _{k \rightarrow+\infty} \mathcal{H}^{1}\left(\widetilde{K}_{k}^{i}\right),
$$

for every $i$. Considering (3.15), we have

$$
\mathcal{H}^{1}(\Gamma) \leq \liminf _{k \rightarrow+\infty} \mathcal{H}^{1}\left(\Gamma_{k}\right)=\liminf _{k \rightarrow+\infty} \sum_{\substack{i=1 \\ \mathcal{H}^{1}\left(\hat{K}^{i}\right)=0}}^{N} \mathcal{H}^{1}\left(\widetilde{K}_{k}^{i}\right)=\sum_{i=1}^{N} \mathcal{H}^{1}\left(\hat{K}^{i}\right)=\mathcal{H}^{1}(\Gamma) .
$$

Since (3.16) holds, in the above relation all liminf are actually limits, so that we obtain the thesis.

Proof of Theorem 3.8. Let $\left(\Gamma_{k}\right)_{k}$ be a sequence in $\mathcal{S}$.

We assume that for each $k$ the set $\Gamma_{k}$ is connected; the general case can be obtained with similar arguments. Hence $\Gamma_{k}$ has the form (3.3):

$$
\Gamma_{k}=\bigcup_{j=1}^{N_{k}} \widetilde{K}_{k}^{j}
$$

Being $N_{k} \leq M$ for every $k$ (see Lemma 3.9), without loss of generality we can assume that $N_{k}=N^{\prime}$ for all $k$. By Blaschke compactness Theorem (see [5, Theorem 4.4.15], up to subsequences $\Gamma_{k} \stackrel{\mathcal{H}}{\longrightarrow} \Gamma$ for a compact connected set $\Gamma$. It remains to prove that $\Gamma \in \mathcal{S}$.

Lemma 3.14 and Condition 3.6.(iii) imply that $\mathcal{H}^{1}\left(\Gamma_{k}\right) \rightarrow \mathcal{H}^{1}(\Gamma)$; therefore $\lambda \leq$ $\mathcal{H}^{1}(\Gamma)<+\infty$, i.e. Condition 3.6.(iii) for $\Gamma$.

Applying again Blaschke's Theorem, up to relabeling the $\widetilde{K}_{k}^{j}$, we can assume $\widetilde{K}_{k}^{j} \stackrel{\mathcal{H}}{\longrightarrow} \hat{K}^{j}$ for some compact set $\hat{K}^{j}$, for $j=1, \ldots, N^{\prime}$; of course, $\Gamma=\hat{K}^{1} \cup \ldots \cup$ $\hat{K}^{N^{\prime}}$. By Proposition 3.2.(v)

$$
\mathcal{H}^{1}\left(\hat{K}^{j}\right)=\lim _{k \rightarrow+\infty} \mathcal{H}^{1}\left(\widetilde{K}_{k}^{j}\right)
$$

and we relabel the sets $\hat{K}^{j}$ so that $\mathcal{H}^{1}\left(\hat{K}^{j}\right)>0$ for $j=1, \ldots, N^{\prime \prime}$ for some $N^{\prime \prime} \leq N^{\prime}$ and $\mathcal{H}^{1}\left(\hat{K}^{j}\right)=0$ for $j=N^{\prime \prime}+1, \ldots, N^{\prime}$ (in this case $\hat{K}^{j}=\left\{x_{j}\right\}$ ). Proposition 3.2 implies also that

$$
\hat{K}^{j} \in \mathcal{R}_{\eta}
$$


for $j=1, \ldots, N^{\prime \prime}$. Being $\Gamma$ and $\hat{K}^{j}$ connected,

$$
\Gamma=\bigcup_{j=1}^{N^{\prime \prime}} \hat{K}^{j} .
$$

Thanks to (3.17) and (3.18), $\Gamma$ can be described as a finite union of $C^{1,1}$ curves in $\mathcal{R}_{\eta}$. We write

$$
\Gamma=\bigcup_{i=1}^{N} \widetilde{K}^{i}
$$

is such a way that Conditions (3.3).(i)-(3.3).(iv) in (3.3) are satisfied. We are left to check Conditions (3.3).(v) and (3.3).(vi). Firstly we remark that, by Lemma 3.13 and Remark 3.3, if $\hat{K}^{j} \cap \hat{K}^{l} \neq \varnothing$, then they intersect at most in their endpoints. Hence for every $i=1, \ldots, N$ it is

$$
\widetilde{K}^{i}=\bigcup_{j \in I_{i}} \hat{K}^{j}
$$

for a set of indices $I_{i} \subset\left\{1, \ldots, N^{\prime}\right\}$.

Assume that $\widetilde{K}^{1}$ satisfies the assumptions in (3.3).(v). If $\widetilde{K}^{1}=\hat{K}^{j}$ for some $j$, then Lemma 3.13 implies that $\mathcal{P}_{2}\left(\widetilde{K}^{1}\right) \cap \hat{K}^{l}=\mathcal{P}_{2}\left(\hat{K}^{j}\right) \cap \hat{K}^{l}=\varnothing$ for every $l \neq j$. Therefore $\mathcal{P}_{2}\left(\widetilde{K}^{1}\right) \cap \widetilde{K}^{h}=\varnothing$ for all $h=2, \ldots N$, which is exactly condition (3.3).(v).

Assume now that, up to relabel the $\hat{K}^{j}$, it is

$$
\widetilde{K}^{1}=\hat{K}^{1} \cup \ldots \cup \hat{K}^{l}
$$

with $\hat{K}^{j} \cap \hat{K}^{j+1}=\left\{y^{j}\right\}$, and that for the remaining $\hat{K}^{j}$ it is

$$
\bigcup_{j=l+1}^{N^{\prime \prime}} \hat{K}^{j}=\bigcup_{i=2}^{N} \widetilde{K}^{i} .
$$

Necessarily, for every $k$ large enough the set $\widetilde{K}_{k}^{1} \cup \ldots \cup \widetilde{K}_{k}^{l}$ is connected and $\widetilde{K}_{k}^{j} \cap$ $\widetilde{K}_{k}^{j+1}=\left\{y_{k}^{j}\right\}$, with

$$
\widetilde{K}_{k}^{1}, \ldots, \widetilde{K}_{k}^{l} \in I_{2}\left(\Gamma_{k}\right),
$$

and $I_{2}\left(\Gamma_{k}\right)$ defined in (3.4). This claim is consequence of Lemma 3.11 and the fact that $\mathcal{H}^{1}\left(\widetilde{K}_{k}^{j}\right) \geq \frac{1}{2} \mathcal{H}^{1}\left(\hat{K}^{j}\right)>0$ for $k$ large: by contradiction, if one of the $\widetilde{K}_{k}^{j}$ belongs to $I_{1}\left(\Gamma_{k}\right)$, then Lemma 3.11 implies that a tip remains at a positive distance (independent of $k$ ) from all the other branches, so that the same holds for its Hausdorff limit; as a consequence, $\widetilde{K}^{1}$ cannot belong to $I_{2}(\Gamma)$.

Call $y^{0}$ the endpoint of $\hat{K}^{1}$ not belonging to $\hat{K}^{2}$, and $y^{l}$ the endpoint of $\hat{K}^{l}$ not belonging to $\hat{K}^{l-1}$. We have $\mathcal{H}^{1}\left(\hat{K}^{j}\right) \geq\left|y^{j-1}-y^{j}\right| \geq \delta$ for $j=1, \ldots, l$, since $y_{k}^{i} \rightarrow y^{i}$ and $\left|y_{k}^{i}-y_{k}^{h}\right| \geq \delta$ for $0 \leq i<h \leq l$. This remark on the lengths, the assumption (3.5) on $\delta$ and the comments in Remark 3.7 imply

$$
\mathcal{P}_{2}\left(\widetilde{K}^{1}\right)=\mathcal{P}_{1}\left(\hat{K}^{1}, y^{0}\right) \cup\left(\hat{K}^{2}\right)_{\beta} \cup \ldots\left(\hat{K}^{l-1}\right)_{\beta} \cup \mathcal{P}_{1}\left(\hat{K}^{l}, y^{l}\right),
$$

where $\left(\hat{K}^{j}\right)_{\beta}=\left\{x \in \Omega: \operatorname{dist}\left(x, \hat{K}^{j}\right)<\beta\right\}$. In particular $\mathcal{P}_{2}\left(\widetilde{K}^{1}\right) \supset \mathcal{P}_{2}\left(\hat{K}^{1}\right) \cup \ldots \cup$ $\mathcal{P}_{2}\left(\hat{K}^{l}\right)$. 
To prove condition (3.3).(v) we argue by contradiction. Assume that $\mathcal{P}_{2}\left(\widetilde{K}^{1}\right) \cap$ $\widetilde{K}^{i} \neq \varnothing$ for some $i \in\{2, \ldots, N\}$; since by Lemma 3.13 and (3.19) it is $\mathcal{P}_{2}\left(\hat{K}^{h}\right) \cap$ $\hat{K}^{m}=\varnothing$ for all $h=1, \ldots l$ and $m \neq l$, there exists

$$
x \in \widetilde{K}^{i} \cap \mathcal{P}_{2}\left(\widetilde{K}^{1}\right) \backslash\left(\mathcal{P}_{2}\left(\hat{K}^{1}\right) \cup \ldots \cup \mathcal{P}_{2}\left(\hat{K}^{l}\right)\right) .
$$

More precisely,

$$
x \in\left(\hat{K}^{j}\right)_{\beta} \backslash \mathcal{P}_{2}\left(\hat{K}^{j}\right)
$$

for some $j \in\{1 \ldots, l\}$ and $x \in \hat{K}^{m}$ for some $m>l$, with $\hat{K}^{m}$ having endpoints $y^{m-1}, y^{m}$. Let $\gamma^{j}$ be the arc-length parametrization of $\hat{K}^{j}$; then

$$
x=\gamma^{j}(s)+z \dot{\gamma}^{j}(s)^{\perp}
$$

for some $|z|<\beta$ and, because of (3.21),

$$
\text { either } s \in[0, \beta / \tan \theta) \quad \text { or } \quad s \in\left(\mathcal{H}^{1}\left(\hat{K}^{j}\right)-\beta / \tan \theta, \mathcal{H}^{1}\left(\hat{K}^{j}\right)\right] .
$$

Assume $(3.23)_{1}$ and remember that $y^{m-1}=\gamma^{j}(0)$ and $y^{j}=\gamma^{j}\left(\mathcal{H}^{1}\left(\hat{\Gamma}^{j}\right)\right)$. Then

$$
\begin{aligned}
\min \left\{\left|x-y^{m-1}\right|,\left|x-y^{m}\right|\right\} \geq & \min \left\{\left|y^{m-1}-y^{j-1}\right|,\left|y^{m}-y^{j-1}\right|\right\}-\left|y^{j-1}-x\right| \\
= & \min \left\{\left|y^{m-1}-y^{j-1}\right|,\left|y^{m}-y^{j-1}\right|\right\} \\
& -\left|\gamma^{j}(0)-\gamma^{j}(s)+z \dot{\gamma}^{j}(s)^{\perp}\right| \\
\geq & \delta-(|z|+s) \geq \delta-\beta-\frac{\beta}{\tan \theta} \\
\geq & \beta\left(\frac{2}{\tan \theta}-1\right)-\beta-\frac{\beta}{\tan \theta}=\frac{\beta}{\tan \theta} .
\end{aligned}
$$

If $(3.23)_{2}$ holds, then by substituting $y^{j-1}$ with $y^{j}$ in the above chain of inequalities we get

$$
\min \left\{\left|x-y^{m-1}\right|,\left|x-y^{m}\right|\right\} \geq \frac{\beta}{\tan \theta} .
$$

Hence $x=\gamma^{m}(s)$ with $s \in\left[\beta / \tan \theta, \mathcal{H}^{1}\left(\hat{\Gamma}^{m}\right)-\beta / \tan \theta\right]$; then, by choice of $\delta$ (see Remark 3.7), this condition implies that $\operatorname{dist}\left(x, \hat{K}^{i}\right) \geq \beta$ for every $i \neq m$, in contradiction to (3.22), since $|z|<\beta$.

In order to check Condition (3.3) (vi) one argues similarly. Instead of (3.20), one has to observe that

$$
\mathcal{P}_{1}\left(\widetilde{K}^{1}, y^{0}\right)=\mathcal{P}_{1}\left(\hat{K}^{1}, y^{0}\right) \cup\left(\hat{K}^{2}\right)_{\beta} \cup \ldots \cup\left(\hat{K}^{l}\right)_{\beta} .
$$

This concludes the proof when the sets $\Gamma_{k}$ are connected.

In conclusion, we prove some further geometrical results which will be useful in the following sections.

Lemma 3.15. Let $\Gamma_{k}, \Gamma \in \mathcal{S}$ and $\Gamma_{k} \stackrel{\mathcal{H}}{\longrightarrow} \Gamma$. Then for every $p \in T_{\Gamma}$ there exists a sequence $\left(p_{k}\right)_{k}$, with $p_{k} \in T_{\Gamma_{k}}$, such that $p_{k} \rightarrow p$.

Proof. By contradiction, assume that there exists $r>0$ such that

$$
\operatorname{dist}\left(p, T_{\Gamma_{k}}\right) \geq r
$$

for every $k$. Without loss of generality, we can assume that $r \in(0, \eta)$ and $r$ satisfies the properties defining a crack tip. Define $K:=\Gamma \cap \overline{B_{r}(p)} \in \mathcal{R}_{\eta}$ and notice that $K \cap \partial B_{r}(p)=\{y\}$. 
Two cases are possible: either

$$
\operatorname{dist}\left(p, S_{\Gamma_{k}}\right) \geq r
$$

for every $k$ (possibly by replacing the previous $r$ with a smaller one), or there exists a sequence $x_{k} \in S_{\Gamma_{k}}$ such that

$$
x_{k} \rightarrow p .
$$

If (3.25) holds, by Hausdorff convergence there exists $x_{k} \rightarrow p$ with $x_{k} \in \Gamma_{k} \backslash\left(S_{\Gamma_{k}} \cup\right.$ $\left.T_{\Gamma_{k}}\right)$. Let $K_{k}$ be the branch of $\Gamma_{k}$ containing $x_{k} ; K_{k} \in \mathcal{R}_{\eta}$. Set $\widetilde{K}_{k}:=K_{k} \cap \overline{B_{r}(p)}$, then $\widetilde{K}_{k} \in \mathcal{R}_{\eta}$. Fix $\varepsilon \in(0, \eta / 2)$; by Hausdorff convergence, for $k$ sufficiently large $\widetilde{K}_{k}:=K_{k} \cap \overline{B_{r}(p)} \subset(K)_{\varepsilon}:=\{x \in \Omega: \operatorname{dist}(x, K)<\varepsilon\}$, hence Condition 3.1.(ii) in Definition 3.1 is not satisfied, in contradiction to the fact that $\widetilde{K}_{k} \in \mathcal{R}_{\eta}$.

If (3.26) is the case, then there exist at least two branches $K_{k}^{1}$ and $K_{k}^{2}$ of $\Gamma_{k}$ containing $x_{k}$ and such that $K_{k}^{i} \backslash \overline{B_{r}(p)} \neq \varnothing$ (because we are assuming (3.24)); let $y_{k}^{i} \in K_{k}^{i} \cap \partial B_{r}(p)$. For $k$ sufficiently large we can assume that $\left|x_{k}-p\right|<r / 2$, so that

$$
\mathcal{H}^{1}\left(K_{k}^{i} \cap \partial B_{r}(p)\right) \geq\left|x_{k}-y_{k}^{i}\right| \geq r / 2
$$

By Lemma 3.11, it must be

$$
\left|y_{k}^{1}-y_{k}^{2}\right| \geq \rho(r / 2) .
$$

Taken $\varepsilon<\frac{1}{2} \min \{\rho(r / 2), r / 2\}$, by Hausdorff convergence we have

$$
K_{k}^{i} \cap \overline{B_{r}(p)} \subset(K)_{\varepsilon} \quad \text { and } \quad\left|y_{k}^{i}-y\right|<\varepsilon,
$$

which imply that $\left|y_{k}^{1}-y_{k}^{2}\right|<2 \varepsilon<\rho(r / 2)$, in contradiction to (3.27).

Lemma 3.16. Let $\Gamma, \Gamma_{k} \in \mathcal{S}$ and $\Gamma_{k} \stackrel{\mathcal{H}}{\longrightarrow} \Gamma$. Assume that for a tip $p \in T_{\Gamma}$ there exist $p_{k}^{1}, p_{k}^{2} \in T_{\Gamma_{k}}, p_{k}^{1} \neq p_{k}^{2}$, converging to $p$. Then there exists $y_{k} \in S_{\Gamma_{k}}$ converging to $p$.

Proof. We argue by contradiction. Assume that

$$
\operatorname{dist}\left(p, S_{\Gamma_{k}}\right) \geq r
$$

for some $r>0$. As in Lemma 3.15, without loss of generality, we can assume that $r \in(0, \eta)$ and $r$ satisfies the properties defining a crack tip. Define $K:=\Gamma \cap \overline{B_{r}(p)} \in$ $\mathcal{R}_{\eta}$.

For $k$ sufficiently large, $\left|p_{k}^{i}-p\right|<r / 2$, so that the connected components $K_{k}^{i}$ containing $p_{k}^{i}$, for $i=1,2$, satisfy the bound from below

$$
\mathcal{H}^{1}\left(K_{k}^{i}\right) \geq r / 2 \text {. }
$$

Then, by Lemma 3.11, it has to be $\left|p_{k}^{1}-p_{k}^{2}\right| \geq \rho(r / 2)$, in contradiction to the fact that $p_{k}^{1}$ and $p_{k}^{2}$ converge both to $p$.

\section{The TIME-INCREMENTAL PROBLEM}

This section is devoted to the study of the discrete-time approximation of the quasistatic evolution. At each incremental step, the fracture is permitted to grow simultaneously at many tips and to develop new branches. In order to avoid nonphysical interactions between them, we need to perform a sort of localization argument to treat each tip separately. This is obtained by keeping trace of the fracture increments at each step in the discrete-time approach. At the end of the section, we establish some a priori estimates and properties of the discrete-time evolutions. 
In the following, given $\Gamma_{1}, \Gamma_{2} \in \mathcal{S}$ with $\Gamma_{1} \subset \Gamma_{2}$, let

$$
\mathcal{C}\left(\Gamma_{1}, \Gamma_{2}\right)
$$

be the set of connected components of $\Gamma_{2} \backslash \Gamma_{1}$. Notice that every element $\mathfrak{c} \in$ $\mathcal{C}\left(\Gamma_{1}, \Gamma_{2}\right)$ is a finite union of $C^{1,1}$ curves. In particular, $\overline{\mathfrak{c}}$ satisfies Conditions (3.3).(i)-(3.3).(vi) in (3.3) and

$$
\mathfrak{c}=\overline{\mathfrak{c}} \backslash \Gamma_{1} .
$$

In addition, if $\overline{\mathfrak{c}^{\prime}} \cap \overline{\mathfrak{c}^{\prime \prime}} \neq \varnothing$ for two distinct components $\mathfrak{c}^{\prime}, \mathfrak{c}^{\prime \prime} \in \mathcal{C}\left(\Gamma_{1}, \Gamma_{2}\right)$, then

$$
\overline{\mathfrak{c}^{\prime}} \cap \overline{\mathfrak{c}^{\prime \prime}} \subset S_{\Gamma_{2}} \cap \Gamma_{1} \text {. }
$$

We now construct the discrete-time evolution with time incremental step $\tau>0$ and initial datum $\left(u_{0}, \Gamma_{0}\right)$ satisfying (2.1). Define

- $u_{\tau}^{0}:=u_{0}$ and $\Gamma_{\tau}^{0}:=\Gamma_{0}$;

- recursively $u_{\tau}^{i}$ and $\Gamma_{\tau}^{i}$ as minimizers of

$$
\|\nabla u\|^{2}+\mathcal{H}^{1}(\Gamma)+\frac{1}{\tau} \sum_{\mathfrak{c} \in \mathcal{C}\left(\Gamma_{\tau}^{i-1}, \Gamma\right)}\left(\mathcal{H}^{1}(\mathfrak{c})\right)^{2}
$$

under the constraints $\Gamma \in \mathcal{S}, \Gamma_{\tau}^{i-1} \subset \Gamma, u \in H^{1}(\Omega \backslash \Gamma)$ and $u=w(i \tau)$ on $\partial_{D} \Omega$.

Proposition 4.1. The minimum problem (4.3) has a solution.

For its proof we need the following lower semicontinuity result and the next stability result about elliptic problems on varying domains.

Lemma 4.2. Consider $\hat{\Gamma}, \Gamma \in \mathcal{S}$ and a sequence $\left(\Gamma_{k}\right)_{k} \subset \mathcal{S}$ such that

$$
\hat{\Gamma} \subset \Gamma_{k} \quad \text { for any } k \text { and } \quad \Gamma_{k} \stackrel{\mathcal{H}}{\longrightarrow} \Gamma \text {. }
$$

Then $\hat{\Gamma} \subset \Gamma$ and

$$
\sum_{\mathfrak{c} \in \mathcal{C}(\hat{\Gamma}, \Gamma)}\left(\mathcal{H}^{1}(\mathfrak{c})\right)^{2} \leq \liminf _{k \rightarrow+\infty} \sum_{\mathfrak{c} \in \mathcal{C}\left(\hat{\Gamma}, \Gamma_{k}\right)}\left(\mathcal{H}^{1}(\mathfrak{c})\right)^{2} .
$$

Proof. The fact that $\hat{\Gamma} \subset \Gamma$ is a direct consequence of (4.4).

We claim that for every $\mathfrak{c} \in \mathcal{C}(\hat{\Gamma}, \Gamma)$ there exists a sequence $\mathfrak{c}_{k} \in \mathcal{C}\left(\hat{\Gamma}, \Gamma_{k}\right)$ such that $\mathfrak{c}_{k} \stackrel{\mathcal{H}}{\longrightarrow} \mathfrak{c}$.

By contradiction, assume that the claim does not hold for some $\mathfrak{c} \in \mathcal{C}(\hat{\Gamma}, \Gamma)$. By (4.4), there exist $\mathfrak{c}_{k}^{1}, \mathfrak{c}_{k}^{2} \in \mathcal{C}\left(\hat{\Gamma}, \Gamma_{k}\right)$ such that

$$
\mathfrak{c}_{k}^{1} \stackrel{\mathcal{H}}{\longrightarrow} \mathfrak{c}^{1} \quad \mathfrak{c}_{k}^{2} \stackrel{\mathcal{H}}{\longrightarrow} \mathfrak{c}^{2}
$$

with $\mathcal{H}^{1}\left(\mathfrak{c}^{1}\right)>0, \mathcal{H}^{1}\left(\mathfrak{c}^{2}\right)>0, \mathfrak{c}^{1} \cup \mathfrak{c}^{2} \subset \mathfrak{c}$ and $\mathfrak{c}^{1} \cup \mathfrak{c}^{2}$ connected. The set $\overline{\mathfrak{c}}^{1} \cap \overline{\mathfrak{c}}^{2}$ contains finitely many points. Notice that, being $\mathfrak{c}^{1} \cup \mathfrak{c}^{2}$ connected and $\left(\mathfrak{c}^{1} \cup \mathfrak{c}^{2}\right) \cap \hat{\Gamma}=$ $\varnothing$, there exists $x \in\left(\overline{\mathfrak{c}}^{1} \cap \overline{\mathfrak{c}}^{2}\right) \backslash \hat{\Gamma}$. In particular, by (4.1) it is $x \in \mathfrak{c}^{1} \cap \mathfrak{c}^{2}$.

By Hausdorff convergence, there exist $x_{k}^{1} \in \mathfrak{c}_{k}^{1}, x_{k}^{2} \in \mathfrak{c}_{k}^{2}$ with

$$
x_{k}^{1}, x_{k}^{2} \rightarrow x .
$$

By assumption (3.7) for the singular points of sets in $\mathcal{S}$, up to subsequences we can assume that either

$$
x_{k}^{1} \in \Gamma_{k} \backslash S_{\Gamma_{k}}
$$


for every $k$ and there exists $C>0$ such that

$$
\operatorname{dist}\left(x_{k}^{1}, S_{\Gamma_{k}} \cap \overline{\mathfrak{c}}_{k}^{1}\right) \geq C,
$$

or the analogous properties hold true for $x_{k}^{2}$ and $\mathfrak{c}_{k}^{2}$. Indeed,

- if both $x_{k}^{1}, x_{k}^{2} \in S_{\Gamma_{k}}$, then (3.7) is contradicted, because $x_{k}^{1} \neq x_{k}^{2}$ and $\mid x_{k}^{1}-$ $x_{k}^{2} \mid \rightarrow 0$.

- If both

$$
\operatorname{dist}\left(x_{k}^{1}, S_{\Gamma_{k}} \cap \overline{\mathfrak{c}}_{k}^{1}\right), \operatorname{dist}\left(x_{k}^{2}, S_{\Gamma_{k}} \cap \overline{\mathfrak{c}}_{k}^{2}\right) \rightarrow 0,
$$

let $y_{k}^{1} \in S_{\Gamma_{k}} \cap \overline{\mathfrak{c}}_{k}^{1}$ with $\left|x_{k}^{1}-y_{k}^{1}\right|=\operatorname{dist}\left(x_{k}^{1}, S_{\Gamma_{k}} \cap \overline{\mathfrak{c}}_{k}^{1}\right)$, and the same for $y_{k}^{2}$. Notice that $y_{k}^{1}, y_{k}^{2} \rightarrow x$.

If $y_{k}^{1}=y_{k}^{2}=: y_{k}$, then $y_{k} \in \overline{\mathfrak{c}}_{k}^{1} \cap \overline{\mathfrak{c}}_{k}^{2}$ and, by (4.2) with $\Gamma_{1}=\hat{\Gamma}$, it is $y_{k} \in \hat{\Gamma}$. Hence $x \in \hat{\Gamma}$, in contradiction to the choice of $x$.

If $y_{k}^{1} \neq y_{k}^{2}$, then (3.7) is contradicted by the fact that $\left|y_{k}^{1}-y_{k}^{2}\right| \rightarrow 0$.

Assume (4.6) and (4.7). Let $\widetilde{K}_{k}$ be the branch in $\Gamma_{k}$ containing $x_{k}^{1}$ and $\gamma_{k}$ its arc-length parametrization. Then $x_{k}^{1}=\gamma_{k}\left(s_{k}\right)$ for some $s_{k} \in\left(0, \mathcal{H}^{1}\left(\widetilde{K}_{k}\right)\right)$; by (4.7), necessarily $s_{k} \geq C$. Hence Lemma 3.11 implies that

$$
\operatorname{dist}\left(x_{k}^{1}, \widetilde{K}_{k}^{\prime}\right) \geq \rho(C)
$$

for all branches $\widetilde{K}_{k}^{\prime}$ different than $\widetilde{K}_{k}$. In particular,

$$
\left|x_{k}^{1}-x_{k}^{2}\right| \geq \rho(C)
$$

for every $k$, in contradiction to (4.5).

To conclude, let $\mathcal{C}(\hat{\Gamma}, \Gamma)=\left\{\mathfrak{c}^{1}, \ldots, \mathfrak{c}^{m}\right\}$ and consider $\mathfrak{c}_{k}^{i} \in \mathcal{C}\left(\hat{\Gamma}, \Gamma_{k}\right)$ such that $\mathfrak{c}_{k}^{i} \stackrel{\mathcal{H}}{\longrightarrow} \mathfrak{c}^{i}$, which exist for what we just proved. Then, by the fact that all components $\overline{\mathfrak{c}}^{i}, \overline{\mathfrak{c}}_{k}^{i}$ satisfy Conditions (3.3).(i)-(3.3).(vi), we can apply Lemma 3.14 to get $\mathcal{H}^{1}\left(\mathfrak{c}_{k}^{i}\right) \rightarrow \mathcal{H}^{1}\left(\mathfrak{c}^{i}\right)$ for $i=1, \ldots, m$. Finally

$$
\begin{aligned}
\sum_{\mathfrak{c} \in \mathcal{C}(\hat{\Gamma}, \Gamma)}\left(\mathcal{H}^{1}(\mathfrak{c})\right)^{2} & =\sum_{i=1}^{m}\left(\mathcal{H}^{1}\left(\mathfrak{c}^{i}\right)\right)^{2}=\lim _{k \rightarrow+\infty} \sum_{i=1}^{m}\left(\mathcal{H}^{1}\left(\mathfrak{c}_{k}^{i}\right)\right)^{2} \\
& \leq \liminf _{k \rightarrow+\infty} \sum_{\mathfrak{c} \in \mathcal{C}\left(\hat{\Gamma}, \Gamma_{k}\right)}\left(\mathcal{H}^{1}(\mathfrak{c})\right)^{2}
\end{aligned}
$$

The following stability result has been proved by several authors in different settings: for Dirichlet problems [33], for Neumann problems [8, 9, 7], in the mixed case [17, Theorem 5.1] (as reported below).

Lemma 4.3. Let $\left(\Gamma_{k}\right)_{k} \subset \mathcal{S}$ be a sequence converging to $\Gamma$ in the Hausdorff metric. Let $\left(g_{k}\right)_{k} \subset H^{1}(\Omega)$ be a sequence converging to $g \in H^{1}(\Omega)$ strongly in $H^{1}(\Omega)$. Let $u_{k} \in H^{1}\left(\Omega \backslash \Gamma_{k}\right)$ and $\hat{u} \in H^{1}(\Omega \backslash \Gamma)$ be the solutions to the elliptic problems

$$
\left\{\begin{array} { l l } 
{ \Delta u _ { k } = 0 } & { \text { in } \Omega \backslash \Gamma _ { k } } \\
{ \frac { \partial u _ { k } } { \partial \nu } = 0 } & { \text { on } \Gamma _ { k } \cup \partial \Omega \backslash \partial _ { D } \Omega } \\
{ u _ { k } = g _ { k } } & { \text { on } \partial _ { D } \Omega }
\end{array} \quad \text { and } \quad \left\{\begin{array}{ll}
\Delta \hat{u}=0 & \text { in } \Omega \backslash \Gamma \\
\frac{\partial \hat{u}}{\partial \nu}=0 & \text { on } \Gamma \cup \partial \Omega \backslash \partial_{D} \Omega \\
\hat{u}=g & \text { on } \partial_{D} \Omega
\end{array}\right.\right.
$$

respectively. Then $\nabla u_{k} \rightarrow \nabla \hat{u}$ strongly in $L^{2}\left(\Omega ; \mathbb{R}^{2}\right)$. 
Proof of Proposition 4.1. Fix $i \in\left\{1, \ldots, N_{\tau}\right\}$. For any $\Gamma \in \mathcal{S}$ let $u_{\Gamma}$ be the minimizer of

$$
\min \left\{\|\nabla v\|^{2}: v \in H^{1}(\Omega \backslash \Gamma) \text { with } v=w(i \tau) \text { on } \partial_{D} \Omega\right\} .
$$

Consider a minimizing sequence $\Gamma_{k} \in \mathcal{S}$ for (4.3). By compactness of the class $\mathcal{S}$, there exist a subsequence, not relabelled, and an element $\widetilde{\Gamma} \in \mathcal{S}$ such that $\Gamma_{k} \stackrel{\mathcal{H}}{\longrightarrow} \widetilde{\Gamma}$. Lemma 3.14 implies that

$$
\mathcal{H}^{1}\left(\Gamma_{k}\right) \rightarrow \mathcal{H}^{1}(\widetilde{\Gamma}) .
$$

Since $\Gamma_{\tau}^{i-1} \subset \Gamma_{k}$ for every $k$ and Lemma 4.2 holds, it follows that $\Gamma_{\tau}^{i-1} \subset \widetilde{\Gamma}$ and

$$
\sum_{\mathfrak{c} \in \mathcal{C}\left(\Gamma_{\tau}^{i-1}, \widetilde{\Gamma}\right)}\left(\mathcal{H}^{1}(\mathfrak{c})\right)^{2} \leq \liminf _{k \rightarrow+\infty} \sum_{\mathfrak{c} \in \mathcal{C}\left(\Gamma_{\tau}^{i-1}, \Gamma_{k}\right)}\left(\mathcal{H}^{1}(\mathfrak{c})\right)^{2} .
$$

Applying Lemma 4.3 we obtain that $\nabla u_{\Gamma_{k}} \rightarrow \nabla u_{\widetilde{\Gamma}}$ strongly in $L^{2}\left(\Omega ; \mathbb{R}^{2}\right)$.

Collecting together (4.8), (4.9) and the last fact, we obtain

$$
\begin{aligned}
& \left\|\nabla u_{\widetilde{\Gamma}}\right\|^{2}+\mathcal{H}^{1}(\widetilde{\Gamma})+\frac{1}{\tau} \sum_{\mathfrak{c} \in \mathcal{C}\left(\Gamma_{\tau}^{i-1}, \widetilde{\Gamma}\right)}\left(\mathcal{H}^{1}(\mathfrak{c})\right)^{2} \\
\leq & \lim _{k \rightarrow+\infty}\left(\left\|\nabla u_{\Gamma_{k}}\right\|^{2}+\mathcal{H}^{1}\left(\Gamma_{k}\right)\right)+\frac{1}{\tau} \liminf _{k \rightarrow+\infty} \sum_{\mathfrak{c} \in \mathcal{C}\left(\Gamma_{\tau}^{i-1}, \Gamma_{k}\right)}\left(\mathcal{H}^{1}(\mathfrak{c})\right)^{2} \\
\leq & \inf \left\{\|\nabla u\|^{2}+\mathcal{H}^{1}(\Gamma)+\frac{1}{\tau} \sum_{\mathfrak{c} \in \mathcal{C}\left(\Gamma_{\tau}^{i-1}, \Gamma\right)}\left(\mathcal{H}^{1}(\mathfrak{c})\right)^{2}\right\},
\end{aligned}
$$

i.e. the couple $\left(u_{\widetilde{\Gamma}}, \widetilde{\Gamma}\right)$ minimizes (4.3) (notice that, by definition of $u_{\widetilde{\Gamma}}$, it is $u_{\widetilde{\Gamma}}=w(i \tau)$ on $\left.\partial_{D} \Omega\right)$.

We introduce the following functions:

- the piecewise-constant interpolant for the displacement $u_{\tau}:[0,1] \rightarrow L^{2}(\Omega)$ as

$$
u_{\tau}(t):=u_{\tau}^{i}
$$

for $i \tau \leq t<(i+1) \tau, i=0, \ldots, N_{\tau}-1$, and $u_{\tau}(t):=u_{\tau}^{N_{\tau}}$ for $\tau N_{\tau} \leq t \leq 1$;

- the piecewise-constant interpolant for the crack set $\Gamma_{\tau}:[0,1] \rightarrow \mathcal{S}$ as

$$
\Gamma_{\tau}(t):=\Gamma_{\tau}^{i}
$$

for $i \tau \leq t<(i+1) \tau, i=0, \ldots, N_{\tau}$, and $\Gamma_{\tau}(t):=\Gamma_{\tau}^{N_{\tau}}$ for $\tau N_{\tau} \leq t \leq 1$;

- the piecewise-constant and piecewise-affine interpolants of the fracture length $\ell_{\tau}, \tilde{\ell}_{\tau}:[0,1] \rightarrow \mathbb{R}$ as

$$
\ell_{\tau}(t):=\mathcal{H}^{1}\left(\Gamma_{\tau}^{i}\right) \quad \text { and } \quad \tilde{\ell}_{\tau}(t):=\mathcal{H}^{1}\left(\Gamma_{\tau}^{i}\right)+\frac{t-i \tau}{\tau} \mathcal{H}^{1}\left(\Gamma_{\tau}^{i+1} \backslash \Gamma_{\tau}^{i}\right)
$$

for $i \tau \leq t<(i+1) \tau, i=0, \ldots, N_{\tau}$, and $\ell_{\tau}(t)=\tilde{\ell}_{\tau}(t):=\mathcal{H}^{1}\left(\Gamma_{\tau}^{N_{\tau}}\right)$ for $\tau N_{\tau} \leq t \leq 1$.

Notice that $u_{\tau}(t)$ solves the problem

$$
\begin{cases}\Delta u_{\tau}(t)=0 & \text { in } \Omega \backslash \Gamma_{\tau}(t) \\ \frac{\partial u_{\tau}(t)}{\partial \nu}=0 & \text { on } \Gamma_{\tau}(t) \cup \partial \Omega \backslash \partial_{D} \Omega \\ u_{\tau}(t)=w_{\tau}(t) & \text { on } \partial_{D} \Omega\end{cases}
$$


with $w_{\tau}(t):=w(i \tau)$ for $i \tau \leq t<(i+1) \tau$, and

$$
\tilde{\ell}_{\tau}(t):=\mathcal{H}^{1}\left(\Gamma_{\tau}^{i}\right)+\frac{t-i \tau}{\tau} \sum_{\mathfrak{c} \in \mathcal{C}\left(\Gamma_{\tau}^{i}, \Gamma_{\tau}^{i+1}\right)} \mathcal{H}^{1}(\mathfrak{c}) .
$$

Remark 4.4. To be precise, by construction $u_{\tau}(t) \in H^{1}\left(\Omega \backslash \Gamma_{\tau}(t)\right)$. Since $\mathcal{L}^{2}\left(\Gamma_{\tau}(t)\right)=$ 0 , sometimes we will consider $u_{\tau}$ as a map in $L^{2}(\Omega)$. Similarly, we will write $\nabla u_{\tau}(t) \in L^{2}\left(\Omega ; \mathbb{R}^{2}\right)$; notice that $\nabla u_{\tau}(t)$ is the distributional gradient of $u_{\tau}(t)$ only on $\Omega \backslash \Gamma_{\tau}(t)$ but, in general, it does not coincide in $\Omega$ with the gradient of an extension of $u_{\tau}(t)$.

Since $w \in H^{1}\left(0,1 ; H^{1}(\Omega)\right)$, for any $0 \leq a<b \leq 1$ it is

$$
w(b)-w(a)=\int_{a}^{b} \dot{w}(t) d t \quad \text { and } \nabla w(b)-\nabla w(a)=\int_{a}^{b} \nabla \dot{w}(t) d t,
$$

where the integrals are Bochner integrals (see [1]). It is also true that

$$
\left\|\int_{a}^{b} \dot{w}(t) d t\right\| \leq \int_{a}^{b}\|\dot{w}(t)\| d t \quad \text { and } \quad\left\|\int_{a}^{b} \nabla \dot{w}(t) d t\right\| \leq \int_{a}^{b}\|\nabla \dot{w}(t)\| d t,
$$

which will be used below.

Proposition 4.5. There exists a bounded non-negative function $\varpi:(0,1) \rightarrow$ $[0,+\infty)$ such that $\varpi(\tau) \rightarrow 0$ as $\tau \rightarrow 0$ and for any $0 \leq i<j \leq N_{\tau}$ the following inequality holds:

$$
\begin{gathered}
\left\|\nabla u_{\tau}^{j}\right\|^{2}+\mathcal{H}^{1}\left(\Gamma_{\tau}^{j}\right)+\frac{1}{\tau} \sum_{h=i}^{j-1} \sum_{\mathfrak{c} \in \mathcal{C}\left(\Gamma_{\tau}^{h}, \Gamma_{\tau}^{h+1}\right)}\left(\mathcal{H}^{1}(\mathfrak{c})\right)^{2} \\
\leq\left\|\nabla u_{\tau}^{i}\right\|^{2}+\mathcal{H}^{1}\left(\Gamma_{\tau}^{i}\right)+2 \int_{i \tau}^{j \tau}\left\langle\nabla u_{\tau}(t), \nabla \dot{w}(t)\right\rangle d t+\varpi(\tau) .
\end{gathered}
$$

Proof. Consider the function $u=u_{\tau}^{h}+w((h+1) \tau)-w(h \tau)$. Since $u \in H^{1}\left(\Omega \backslash \Gamma_{\tau}^{h}\right)$ and $u=w((h+1) \tau)$ on $\partial_{D} \Omega$, the couple $\left(u, \Gamma_{\tau}^{h}\right)$ can be used as competitor in (4.3) at the $h+1$ step. Then

$$
\begin{aligned}
& \left\|\nabla u_{\tau}^{h+1}\right\|^{2}+\mathcal{H}^{1}\left(\Gamma_{\tau}^{h+1}\right)+\frac{1}{\tau} \sum_{\mathfrak{c} \in \mathcal{C}\left(\Gamma_{\tau}^{h}, \Gamma_{\tau}^{h+1}\right)}\left(\mathcal{H}^{1}(\mathfrak{c})\right)^{2} \\
\leq & \left\|\nabla u_{\tau}^{h}+\nabla w((h+1) \tau)-\nabla w(h \tau)\right\|^{2}+\mathcal{H}^{1}\left(\Gamma_{\tau}^{h}\right) \\
= & \left\|\nabla u_{\tau}^{h}\right\|^{2}+2\left\langle\nabla u_{\tau}(t), \nabla w((h+1) \tau)-\nabla w(h \tau)\right\rangle \\
& +\|\nabla w((h+1) \tau)-\nabla w(h \tau)\|^{2}+\mathcal{H}^{1}\left(\Gamma_{\tau}^{h}\right) \\
\leq & \left\|\nabla u_{\tau}^{h}\right\|^{2}+\mathcal{H}^{1}\left(\Gamma_{\tau}^{h}\right)+2 \int_{h \tau}^{(h+1) \tau}\left\langle\nabla u_{\tau}(t), \nabla \dot{w}(t)\right\rangle d t \\
& +\left(\max _{0 \leq n<N_{\tau}} \int_{n \tau}^{(n+1) \tau}\|\nabla \dot{w}(t)\| d t\right) \int_{h \tau}^{(h+1) \tau}\|\nabla \dot{w}(t)\| d t .
\end{aligned}
$$

Iterating over $h=i, \ldots, j-1$ and defining

$$
\varpi(\tau):=\left(\max _{0 \leq n<N_{\tau}} \int_{n \tau}^{(n+1) \tau}\|\nabla \dot{w}(t)\| d t\right) \int_{0}^{1}\|\nabla \dot{w}(t)\| d t,
$$

we obtain the thesis. 
Lemma 4.6. There exists a constant $C>0$, independent of $\tau$ and $t$, such that the following estimates hold true for every $\tau \in(0,1)$ and $t \in[0,1]$ :

$$
\begin{aligned}
& \left\|\nabla u_{\tau}(t)\right\| \leq C \\
& \frac{1}{\tau} \sum_{i=0}^{N_{\tau}-1} \sum_{\mathfrak{c} \in \mathcal{C}\left(\Gamma_{\tau}^{i}, \Gamma_{\tau}^{i+1}\right)}\left(\mathcal{H}^{1}(\mathfrak{c})\right)^{2} \leq C \\
& \mathcal{H}^{1}\left(\Gamma_{\tau}(t)\right) \leq C .
\end{aligned}
$$

Proof. Fix $t \in[0,1]$ and let $j=j(t) \in 0, \ldots, N_{\tau}-1$ be such that it satisfies $j \tau \leq t<(j+1) \tau$. By the inequality in Proposition 4.5 for $i=0$, we obtain

$$
\left\|\nabla u_{\tau}^{j}\right\|^{2}+\frac{1}{\tau} \sum_{i=0}^{j-1} \sum_{\mathfrak{c} \in \mathcal{C}\left(\Gamma_{\tau}^{i}, \Gamma_{\tau}^{i+1}\right)}\left(\mathcal{H}^{1}(\mathfrak{c})\right)^{2} \leq\left\|\nabla u_{0}\right\|^{2}+\int_{0}^{j \tau}\left\langle\nabla u_{\tau}(\xi), \nabla \dot{w}(\xi)\right\rangle d \xi+\varpi(\tau) .
$$

Hölder's inequality and (4.15) imply

$$
\frac{1}{\tau} \sum_{i=0}^{j} \sum_{\mathfrak{c} \in \mathcal{C}\left(\Gamma_{\tau}^{i}, \Gamma_{\tau}^{i+1}\right)}\left(\mathcal{H}^{1}(\mathfrak{c})\right)^{2} \leq C+\left(\int_{0}^{t}\left\|\nabla u_{\tau}(\xi)\right\|^{2} d \xi\right)^{1 / 2}\left(\int_{0}^{t}\|\nabla \dot{w}(\xi)\|^{2} d \xi\right)^{1 / 2}
$$

and

$$
\left\|\nabla u_{\tau}(t)\right\|^{2} \leq C+\left(\int_{0}^{t}\left\|\nabla u_{\tau}(\xi)\right\|^{2} d \xi\right)^{1 / 2}\left(\int_{0}^{t}\|\nabla \dot{w}(\xi)\|^{2} d \xi\right)^{1 / 2},
$$

where $C>0$ is independent of $\tau$ and $t$.

By a refined version of the Gronwall lemma (see [4, Lemma 4.1.8]), we deduce that for every $t \in[0,1]$

$$
\left(\int_{0}^{t}\left\|\nabla u_{\tau}(\xi)\right\|^{2} d \xi\right)^{1 / 2} \leq(C)^{1 / 2}+2\|\nabla \dot{w}\|_{L^{2}\left(0,1 ; L^{2}\left(\Omega ; \mathbb{R}^{2}\right)\right)}
$$

The last two inequalities imply that $\nabla u_{\tau}(t)$ is bounded in $L^{2}\left(\Omega ; \mathbb{R}^{2}\right)$ uniformly with respect to $\tau, t$, i.e. (4.12). Then, considering (4.16) and (4.12), the estimate (4.13) follows.

Finally, Lemma 3.10 implies (4.14).

Lemma 4.7. It is $\tilde{\ell}_{\tau} \in H^{1}(0,1)$ and

$$
\left\|\tilde{\ell}_{\tau}\right\|_{H^{1}(0,1)} \leq C
$$

for every $\tau$, with $C$ independent of $\tau$.

Proof. By Lemma $3.10, \mathcal{H}^{1}(\Gamma) \leq C$ for any $\Gamma \in \mathcal{S}$. Then

$$
0 \leq \tilde{\ell}_{\tau}(t) \leq \mathcal{H}^{1}\left(\Gamma_{\tau}^{i}\right)+\mathcal{H}^{1}\left(\Gamma_{\tau}^{i+1}\right) \leq 2 C
$$

for any $t$ and $\tau$. 
Observe that Lemma 3.9 implies that at each step the set $\mathcal{C}\left(\Gamma_{\tau}^{i}, \Gamma_{\tau}^{i+1}\right)$ contains at most $M$ elements. Then

$$
\begin{aligned}
\int_{0}^{1}\left|\dot{\tilde{l}}_{\tau}(t)\right|^{2} d t & =\sum_{i=0}^{N_{\tau}-1} \int_{i \tau}^{(i+1) \tau}\left|\frac{1}{\tau} \sum_{\mathfrak{c} \in \mathcal{C}\left(\Gamma_{\tau}^{i}, \Gamma_{\tau}^{i+1}\right)} \mathcal{H}^{1}(\mathfrak{c})\right|^{2} d t \\
& \leq 2^{M} \sum_{i=0}^{N_{\tau}-1} \frac{1}{\tau} \sum_{\mathfrak{c} \in \mathcal{C}\left(\Gamma_{\tau}^{i}, \Gamma_{\tau}^{i+1}\right)}\left(\mathcal{H}^{1}(\mathfrak{c})\right)^{2} \leq 2^{M} C
\end{aligned}
$$

where the last inequality is consequence of (4.13).

\section{The continuous-time evolution}

In this section we select a continuous-time evolution $t \mapsto(u(t), \Gamma(t))$ as limit of discrete-time ones, esploiting the a priori estimates of the previous section and compactness results. Among all evolutions $t \mapsto(\widetilde{u}(t), \widetilde{\Gamma}(t)) \in L^{2}(\Omega) \times \mathcal{S}$ with $t \mapsto \widetilde{\Gamma}(t)$ monotone and $\widetilde{u}(t) \in H^{1}(\Omega \backslash \widetilde{\Gamma}(t))$ in static equilibrium with respect to the boundary datum $w(t)$, the above selection provides the evolution $t \mapsto(u(t), \Gamma(t))$ with additional properties, as explained in Section 6.

By construction, the set functions $\Gamma_{\tau}:[0,1] \rightarrow \mathcal{S}$ are monotone increasing (with respect to the inclusion ordering). Considering the version of the Helly's theorem proved in [17, Theorem 6.3], there exists a subsequence (not relabelled) $\Gamma_{\tau}$ and a function $\Gamma:[0,1] \rightarrow 2^{\Omega}$ such that

$$
\Gamma_{\tau}(t) \stackrel{\mathcal{H}}{\longrightarrow} \Gamma(t)
$$

for every $t \in[0,1]$. By the compactness result in Theorem 3.8, it is $\Gamma:[0,1] \rightarrow \mathcal{S}$.

Concerning the displacements $u_{\tau}$, the following convergence result holds. Let $u(t) \in H^{1}(\Omega \backslash \Gamma(t))$ be the solution to

$$
\begin{cases}\Delta u(t)=0 & \text { in } \Omega \backslash \Gamma(t) \\ \frac{\partial u(t)}{\partial \nu}=0 & \text { on } \Gamma(t) \cup \partial \Omega \backslash \partial_{D} \Omega \\ u(t)=w(t) & \text { on } \partial_{D} \Omega .\end{cases}
$$

Since $\Gamma_{\tau}(t) \stackrel{\mathcal{H}}{\longrightarrow} \Gamma(t), w_{\tau}(t) \rightarrow w(t)$ strongly in $H^{1}(\Omega)$, and (4.10) and (5.2) hold, by applying Lemma 4.3 we conclude that

$$
\nabla u_{\tau}(t) \rightarrow \nabla u(t)
$$

strongly in $L^{2}\left(\Omega ; \mathbb{R}^{2}\right)$ for every $t$. Furthermore the bound (4.12) implies

$$
\|\nabla u(t)\| \leq C,
$$

with $C$ independent of $t$.

Now we analize the approximation process, in order to obtain the growth properties of the evolution $t \mapsto(u(t), \Gamma(t))$ announced at the beginning of the section.

Applying the classical Helly's theorem, there exists a subsequence (not relabelled) $\ell_{\tau}$ and a function $\ell:[0,1] \rightarrow \mathbb{R}$ such that

$$
\ell_{\tau}(t) \rightarrow \ell(t)
$$

for every $t \in[0,1]$. By Lemma 3.14 and (5.1), it is $\ell(t)=\mathcal{H}^{1}(\Gamma(t))$. 
Proposition 5.1. The function $\ell$ obtained in (5.5) belongs to $H^{1}(0,1)$. In particular, $\tilde{\ell}_{\tau}(t) \rightarrow \ell(t)$ for $t \in[0,1]$ and $\tilde{\ell}_{\tau} \rightarrow \ell$ weakly in $H^{1}(0,1)$.

Proof. By the uniform bound proved in Lemma 4.7, up to subsequences it is

$$
\tilde{\ell}_{\tau} \rightarrow \tilde{\ell}
$$

weakly in $H^{1}(0,1)$ for some $\tilde{\ell} \in H^{1}(0,1)$, and

$$
\|\tilde{\ell}\|_{H^{1}(0,1)} \leq \liminf _{\tau \rightarrow 0}\left\|\tilde{\ell}_{\tau}\right\|_{H^{1}(0,1)} \leq C .
$$

By definition of $\tilde{\ell}_{\tau}$ and $\ell_{\tau}$, we have

$$
\begin{aligned}
0 \leq \tilde{\ell}_{\tau}(t)-\ell_{\tau}(t) & =\frac{t-i \tau}{\tau} \mathcal{H}^{1}\left(\Gamma_{\tau}^{i+1} \backslash \Gamma_{\tau}^{i}\right) \leq \tau \dot{\tilde{\ell}}_{\tau}(t) \\
& =\int_{i \tau}^{(i+1) \tau} \dot{\tilde{\ell}}_{\tau}(\xi) d \xi \leq \tau^{1 / 2}\left(\int_{i \tau}^{(i+1) \tau}\left|\dot{\tilde{\ell}}_{\tau}(\xi)\right|^{2} d \xi\right)^{1 / 2} \leq \tau^{1 / 2} C,
\end{aligned}
$$

where the last inequality is due to Lemma 4.7 and $i$ is such that $i \tau \leq t<(i+1) \tau$. Then, considering (5.5), as $\tau \rightarrow 0$ we obtain that $\tilde{\ell}_{\tau}(t) \rightarrow \ell(t)$ for $t \in[0,1]$. Finally, by uniqueness of the limit, it is $\tilde{\ell}=\ell$ a.e. in $[0,1]$, so that by $(5.6)$ we conclude.

Corollary 5.2. The set function

$$
\Gamma:[0,1] \rightarrow \mathcal{S}
$$

is continuous with respect to the Hausdorff convergence.

Proof. For any $t \in(0,1)$ define the left- and right-limit of $\Gamma(\cdot)$ at $t$ as

$$
\Gamma^{-}(t):=\overline{\bigcup_{t^{\prime}<t} \Gamma\left(t^{\prime}\right)} \quad \text { and } \quad \Gamma^{+}(t):=\bigcap_{t^{\prime}>t} \Gamma\left(t^{\prime}\right) .
$$

By compactness of $\mathcal{S}$, both limits belong to $\mathcal{S}$ and it is easy to check that $\Gamma^{-}(t) \subset$ $\Gamma^{+}(t)$. Let $t_{n}^{\prime}<t<t_{n}^{\prime \prime}$ be sequences converging to $t$; then, by monotonicity of $\Gamma(\cdot)$, we have

$$
0 \leq \mathcal{H}^{1}\left(\Gamma^{+}(t) \backslash \Gamma^{-}(t)\right) \leq \mathcal{H}^{1}\left(\Gamma\left(t_{n}^{\prime \prime}\right) \backslash \Gamma\left(t_{n}^{\prime}\right)\right)=\ell\left(t_{n}^{\prime \prime}\right)-\ell\left(t_{n}^{\prime}\right) \rightarrow 0,
$$

where the last limit is due to the continuity of $\ell$, as consequence of Proposition 5.1.

If, by contradiction, it is $\Gamma^{-}(t) \neq \Gamma^{+}(t)$, the above discussion implies that

$$
\Gamma^{+}(t)=\Gamma^{-}(t) \cup A(t)
$$

with $A(t)$ totally disconneted (since $\mathcal{H}^{1}(A(t))=0$; see [19, Proposition 2.5]). This contradicts the fact that each connected component of $\Gamma^{+}(t)$ has length at least $\lambda$ (as requested by Definition 3.6.(iii)).

We look at the approximation process in correspondence of the tips of the crack $\Gamma(t)$; the presence of several branches makes the scenario richer.

For simplicity of notation, set

$$
T(t):=T_{\Gamma(t)} \quad \text { and } \quad S(t):=S_{\Gamma(t)}
$$

and

$$
T_{\tau}(t):=T_{\Gamma_{\tau}(t)} \quad \text { and } \quad S_{\tau}(t):=S_{\Gamma_{\tau}(t)} .
$$


For every $t \in(0,1]$ we define

$$
M T(t):=\Gamma(t) \backslash \bigcup_{t^{\prime}<t} \Gamma\left(t^{\prime}\right)
$$

by Corollary 5.2 and the geometric properties of the class $\mathcal{S}$, it is not difficult to prove that

$$
M T(t)=T(t) \backslash \bigcup_{t^{\prime}<t} T\left(t^{\prime}\right),
$$

motivating the notation $M T$ which stands for "moving tips". We call

$$
\mathcal{A}_{0}:=\{t \in(0,1]: M T(t) \neq \varnothing\}
$$

the set of instants when the fracture has really grown, at least at one tip.

We cannot exclude a priori that a tip of the continuous-time evolution is the limit point of (finitely) many tips of the approximating discrete-time evolutions. If this happens, we have some difficulties in characterizing the exact behaviour of the continuous-time process (see the end of Subsection 6.2). Hence below we introduce and describe the properties of a subset $\mathcal{A}$ of $\mathcal{A}_{0}$, containing the instants $t$ such that every moving tip at $t \in \mathcal{A}$ is approximated exactly by one tip of each discretetime evolution. The set $\mathcal{A}$ will play an important role later, in the description of a stability criterion for the continuous-time evolution.

Lemma 5.3. Let $\mathcal{A}$ be the set of instants $t \in \mathcal{A}_{0}$ such that for every $p \in M T(t)$ there exist a neighbourhood $U$ of $p$ and a value $\nu(t, p)>0$ such that for every $\tau \leq \nu(t, p)$ the following two conditions hold:

- $T_{\tau}(t) \cap U$ contains one and only one element, denoted $p_{\tau}(t)$;

- $S_{\tau}(t) \cap U=\varnothing$.

Then $\mathcal{A}_{0} \backslash \mathcal{A}$ is finite.

Proof. By definition of the class $\mathcal{S}$ and Lemma 3.9, the cardinality of $S_{\tau}(1)$ is uniformly bounded with respect to $\tau$. Up to considering a subsequence, we can assume that

$$
S_{\tau}(1)=\left\{x_{\tau}^{1}, \ldots, x_{\tau}^{M}\right\}
$$

and $x_{\tau}^{j} \rightarrow x^{j}$ as $\tau \rightarrow 0$, for $j=1, \ldots, M$. Notice that $\left|x^{j}-x^{l}\right| \geq \delta$ if $j \neq l$, since the same holds for $x_{\tau}^{j}$ and $x_{\tau}^{l}$ (see Condition 3.6.(iv)).

By Proposition 3.15 and since $T(t)$ contains finitely many points (see again Lemma 3.9), for every $t \in[0,1]$ and $r>0$ there exists $\tilde{\nu}(t, r)>0$ such that

$$
B_{r}(p) \cap T_{\tau}(t) \neq \varnothing
$$

for every $p \in T(t)$ and $\tau<\tilde{\nu}(t, r)$.

Let $t \in \mathcal{A}_{0} \backslash \mathcal{A}$. Then there exists $p \in M T(t)$ such that for every $r>0$ and every $\nu \in(0, \tilde{\nu}(t, r))$ there exists $\tau_{\nu}<\nu$ such that $T_{\tau_{\nu}}(t) \cap B_{r}(p)$ has at least two elements or $S_{\tau_{\nu}}(t) \cap B_{r}(p) \neq \varnothing$.

In the first case, by Lemma 3.16 there exists $y_{\tau_{\nu}} \in S_{\tau_{\nu}}(t)$ such that $y_{\tau_{\nu}} \rightarrow p$ as $\nu \rightarrow 0$ (so $\tau_{\nu} \rightarrow 0$ ). Being $S_{\tau_{\nu}}(t) \subset S_{\tau_{\nu}}(1)$, then $y_{\tau_{\nu}}=x_{\tau_{\nu}}^{j_{\nu}}$ for some $j_{\nu} \in$ $\{1, \ldots, M\}$; it follows that $p=x^{j}$ for some $j$.

Similarly, if $S_{\tau_{\nu}}(t) \cap B_{r}(p) \neq \varnothing$ then $p=x^{j}$ for some $j$.

Since $p\left(=x^{j}\right) \notin M T\left(t^{\prime}\right)$ for every $t^{\prime} \in \mathcal{A}_{0} \backslash\{t\}$ (being $M T\left(t^{\prime}\right) \neq M T\left(t^{\prime \prime}\right)$ for any distinct $\left.t^{\prime}, t^{\prime \prime} \in \mathcal{A}_{0}\right)$ and the points $x^{j}$ are a finite number, then also $\mathcal{A}_{0} \backslash \mathcal{A}$ is finite. 
By definition of crack tip, for any fixed $\hat{t} \in(0,1]$ there exists $r_{1}(\hat{t}) \in(0, \eta)$ such that

$$
\overline{B_{r_{1}(\hat{t})}(p)} \cap \Gamma(\hat{t})
$$

is a curve in $\mathcal{R}_{\eta}$ for every $p \in T(\hat{t})$. In addition, $r_{1}(\hat{t})$ can be chosen so that

$$
\begin{aligned}
& \partial B_{r_{1}(\hat{t})}(p) \cap \Gamma(\hat{t})=\{x(\hat{t}, p)\} \\
& \mathcal{H}^{1}\left(B_{r_{1}(\hat{t})}(p) \cap \Gamma(\hat{t})\right) \leq \lambda \\
& \overline{B_{r_{1}(\hat{t})}(p)} \cap S(\hat{t})=\varnothing .
\end{aligned}
$$

It results that the points $p$ and $x(\hat{t}, p)$ belong to the same branch of $\Gamma(\hat{t})$ and $x(\hat{t}, p) \notin S(\hat{t})$. Since the function $\Gamma:[0,1] \rightarrow \mathcal{S}$ is monotone and continuous with respect to the Hausdorff convergence (see Corollary 5.2), and (5.8) holds, for instants $t$ in a left neighbourhood of $\hat{t}$ and for each $p \in T(\hat{t})$ the set $B_{r_{1}(\hat{t})}(p) \cap T(t)$ has exactly one element, labelled $p(t)$, i.e.

$$
B_{r_{1}(\hat{t})}(p) \cap T(t)=\{p(t)\},
$$

and

$$
T(t)=\{p(t): p \in T(\hat{t})\} .
$$

We are able to estimate the size of the left neighbourhood of $\hat{t}$ in which the above conditions hold. Indeed, define

$$
\alpha_{1}(\hat{t}, p):=\inf \{t \in[0, \hat{t}): x(\hat{t}, p) \in \Gamma(t)\},
$$

where $x(\hat{t}, p)$ has been introduced in (5.8). Then we have

$$
\begin{aligned}
r_{1}(\hat{t}) & \leq \mathcal{H}^{1}\left(B_{r_{1}(\hat{t})}(p) \cap \Gamma(\hat{t})\right) \leq \int_{\alpha_{1}(\hat{t}, p)}^{\hat{t}} \dot{\ell}(t) d t \\
& \leq\left(\hat{t}-\alpha_{1}(\hat{t}, p)\right)^{1 / 2}\left(\int_{\alpha_{1}(\hat{t}, p)}|\dot{\ell}(t)|^{2} d t\right)^{1 / 2} \\
& \leq C\left(\hat{t}-\alpha_{1}(\hat{t}, p)\right)^{1 / 2}
\end{aligned}
$$

so that

$$
\hat{t}-\alpha_{1}(\hat{t}, p) \geq C r_{1}(\hat{t})^{2}
$$

with $C$ independent of $\hat{t}$ and $p$.

Since $T(\hat{t})$ contains finitely many points, if we set

$$
\alpha_{1}(\hat{t}):=\max \left\{\alpha_{1}(\hat{t}, p): p \in T(\hat{t})\right\},
$$

then (5.9) and (5.10) hold for every $t \in\left(\alpha_{1}(\hat{t}), \hat{t}\right]$.

Remark 5.4. Notice that we cannot infer anything about the local behaviour of $\Gamma(\cdot)$ at the instants after $\hat{t}$, since new branches might spring out at some tip $p \in T(\hat{t})$.

Lemma 5.5. For every $\hat{t} \in(0,1] \backslash \mathcal{A}_{0}$ there exists $\alpha(\hat{t}) \in\left[\alpha_{1}(\hat{t}), \hat{t}\right)$ such that $\Gamma(t)=$ $\Gamma(\hat{t})$ for every $t \in(\alpha(\hat{t}), \hat{t}]$. In particular $T(t)=T(\hat{t})$ for every $t \in(\alpha(\hat{t}), \hat{t}]$.

Proof. It is a straightforward consequence of the definition of $M T$. 
By definition of $\mathcal{A}$, at instants $t \in \mathcal{A}$ each crack tip is locally approximated by exactly one tip, while singular points of the approximating sequence remain "distant" (see Fig. 4). The following lemma shows that these properties are preserved locally in a left neighbourhood of every instant in $\mathcal{A}$. The importance of this result lies in the fact that this left neighbourhood is not necessarily entirely contained in $\mathcal{A}$.

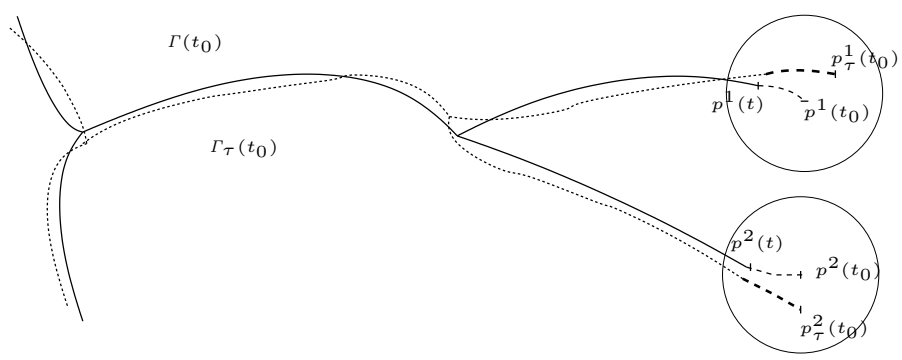

Figure 4: The crack set $\Gamma\left(t_{0}\right)$ and, dotted, a discrete-time approximating crack set $\Gamma_{\tau}\left(t_{0}\right)$ at an instant $t_{0} \in \mathcal{A}$, in correspondence of two tips $p^{1}\left(t_{0}\right), p^{2}\left(t_{0}\right) \in M T\left(t_{0}\right)$.

Lemma 5.6. Let $\hat{t} \in \mathcal{A}$. Then there exist $\alpha(\hat{t}) \in\left[\alpha_{1}(\hat{t}), \hat{t}\right), \nu(\hat{t})>0$ and $r(\hat{t}) \in$ $(0, \eta)$ such that the following facts hold for every $t \in(\alpha(\hat{t}), \hat{t}]$ :

(i) if $p \in T(\hat{t}) \backslash M T(\hat{t})$, then $p \in T(t)$;

(ii) if $p \in M T(\hat{t})$, then for every $\tau<\nu(\hat{t})$ the set $B_{r(\hat{t})}(p) \cap T_{\tau}(t)$ has exactly one element, that we label $p_{\tau}(t)$.

Proof. If $p \in T(\hat{t}) \backslash M T(\hat{t})$, then argue as in Lemma 5.5 and call $\beta_{1}(\hat{t})$ what therein is $\alpha(\hat{t})$.

Consider now $p \in M T(\hat{t})$. By definition of $\mathcal{A}$, there exist $r(\hat{t})>0$ and $\nu_{1}(\hat{t})>0$ such that

$$
T_{\tau}(\hat{t}) \cap B_{r(\hat{t})}(p)=\left\{p_{\tau}(\hat{t})\right\} \quad \text { and } \quad S_{\tau}(\hat{t}) \cap B_{r(\hat{t})}(p)=\varnothing
$$

for every $p \in M T(\hat{t})$. In particular, we can choose $r(\hat{t}) \in\left(0, r_{1}(\hat{t})\right]$, where $r_{1}(\hat{t})$ was introduced in (5.8), and such that

$$
\sup \left\{\mathcal{H}^{1}(K): K \in \mathcal{R}_{\eta}, K \subset \overline{B_{r(\hat{t})}(0)}\right\}<\lambda .
$$

The above conditions on $r(\hat{t})$ imply that

$$
\Gamma_{\tau}(\hat{t}) \cap \overline{B_{r(\hat{t})}(p)} \in \mathcal{R}_{\eta}
$$

and every connected component of $\Gamma_{\tau}(\hat{t})$ is not completely contained in $\overline{B_{r(\hat{t})}(p)}$, because of (5.13) and of the constraint given by Condition 3.6.(iii).

For simplicity of notation, in the remaining of the proof we write

$$
r=r(\hat{t}) \quad \text { and } \quad \nu_{1}=\nu_{1}(\hat{t}) .
$$

Fix $p \in M T(\hat{t})$. Let $\nu=\nu(\hat{t}) \in\left(0, \nu_{1}\right)$ be such that $p_{\tau}(\hat{t}) \in B_{r / 2}(p)$ for every $\tau<\nu$ ( $\operatorname{such} \nu$ exists since, by Proposition 3.15, it is $p_{\tau}(\hat{t}) \rightarrow p$ as $\tau \rightarrow 0$ ); if 
necessary, later we will replace $\nu$ with a smaller one. By (5.12) and (5.13), it follows that

for every $\tau<\nu$.

$$
\mathcal{H}^{1}\left(\Gamma_{\tau}(\hat{t}) \cap B_{r}(p)\right) \geq \frac{r}{2}
$$

Define

$$
t_{\tau}:=\inf \left\{t \in[0, \hat{t}): \Gamma_{\tau}\left(t^{\prime}\right) \cap B_{r}(p) \neq \varnothing \text { for every } t^{\prime} \in(t, \hat{t})\right\} .
$$

If $t_{\tau}=0$ for any $\tau<\nu$, set $\beta_{2}(\hat{t}):=\alpha_{1}(\hat{t})$, where $\alpha_{1}(\hat{t})$ was defined in (5.11). If $t_{\tau}>0$ for some $\tau<\nu$, we argue in the following way: for any $\tau$ let $i_{\tau}, j_{\tau} \in \mathbb{N}$ be such that

$$
i_{\tau} \leq t_{\tau}<\left(i_{\tau}+1\right) \tau \text { and } j_{\tau} \leq \hat{t}<\left(j_{\tau}+1\right) \tau .
$$

By (5.14), Lemma 4.7 and Hölder inequality, we have

$$
\begin{aligned}
\frac{r}{2} & \leq \mathcal{H}^{1}\left(\Gamma_{\tau}(\hat{t}) \backslash \Gamma_{\tau}\left(t_{\tau}\right)\right)=\sum_{h=i_{\tau}}^{j_{\tau}} \sum_{C \in \mathcal{C}\left(\Gamma_{\tau}^{h}, \Gamma_{\tau}^{h+1}\right)} \mathcal{H}^{1}(C)=\int_{i_{\tau} \tau}^{\left(j_{\tau}+1\right) \tau} \dot{\tilde{\ell}}_{\tau}(t) d t \\
& \leq \int_{t_{\tau}-\tau}^{\hat{t}+\tau} \dot{\tilde{\ell}}_{\tau}(t) d t \leq\left(\hat{t}-t_{\tau}+2 \tau\right)^{1 / 2}\left(\int_{0}^{1}\left|\dot{\tilde{\ell}}_{\tau}(t)\right|^{2} d t\right)^{1 / 2} \leq C\left(\hat{t}-t_{\tau}+2 \tau\right)^{1 / 2}
\end{aligned}
$$

with $C>0$ independent of $\tau$ and $\hat{t}$. Define

$$
\beta_{2}(\hat{t}):=\max \left\{\alpha_{1}(\hat{t}), \hat{t}+\nu(\hat{t})-\frac{r^{2}}{4 C^{2}}\right\}
$$

and choose $\nu(\hat{t})$ such that, in addition to being smaller than $\nu_{1}$, it satifies

$$
\nu(\hat{t}) \leq \frac{1}{4} \frac{r^{2}}{4 C^{2}} .
$$

Then $\beta_{2}(\hat{t})<\hat{t}$ and, taking into account (5.15), for every $\tau<\nu(\hat{t})$ it is

$$
t_{\tau} \leq \hat{t}+\tau-\frac{r^{2}}{4 C^{2}} \leq \hat{t}+\nu(\hat{t})-\frac{r^{2}}{4 C^{2}} \leq \beta_{2}(\hat{t}) .
$$

Summarizing, we have shown that for every $\tau<\nu(\hat{t})$ and $t \in\left(\beta_{2}(\hat{t}), \hat{t}\right]$

$$
\Gamma_{\tau}(t) \cap B_{r(\hat{t})}(p) \neq \varnothing \quad \text { and } \quad \overline{\Gamma_{\tau}(t) \cap B_{r(\hat{t})}(p)} \in \mathcal{R}_{\eta} .
$$

Then we can conclude that $T_{\tau}(t) \cap B_{r(\hat{t})}(p)$ has only one element, denoted by $p_{\tau}(t)$.

Since $M T(\hat{t})$ contains finitely many points, we can choose $r(\hat{t}), \beta_{2}(\hat{t})$ and $\nu(\hat{t})$ such that the above property holds for every $p \in M T(\hat{t})$.

Finally, define $\alpha(\hat{t}):=\max \left\{\beta_{1}(\hat{t}), \beta_{2}(\hat{t})\right\}$, so that both (i) and (ii) are valid for $t \in(\alpha(\hat{t}), \hat{t}]$.

5.1. Velocity of the crack tips. In this subsection we introduce the notion of velocity of the front $T(t)$ of the fracture. It will play a role in the dissipative term of the energy and for a Griffith-like stability criterion for the crack growth. We provide two equivalent descriptions, both interesting for different reasons. Firstly we introduce the velocity by means of a distributional approach, based on the theory of absolutely continuous maps with values in the space of bounded measures. This point of view gives a picture of the situation in the whole of $\Omega$, and it somehow 
reminds the approach suggested by [26]. Instead, the second description is local and is based on the parametrization of the branches of the crack.

At the very beginning, we summarize what we know so far about the crack growth $t \mapsto \Gamma(t)$. As observed in Corollary 5.2, the set function $\Gamma(\cdot):[0,1] \rightarrow \mathcal{S}$ is continuous with respect to the Hausdorff convergence. By construction of the class $\mathcal{S}$, it is $\operatorname{card}(S(1)) \leq M$ for some $M \in \mathbb{N}$. Since the map $t \mapsto S(t)$ is monotone increasing with respect to inclusion, there exists a partition of the interval $[0,1]$

$$
0=a_{0}<a_{1}<\ldots<a_{n}=1
$$

such that

- $S(t)=S\left(t^{\prime}\right)$ for every $t, t^{\prime} \in\left(a_{i}, a_{i+1}\right]$;

- $\operatorname{card}(S(t))<\operatorname{card}\left(S\left(t^{\prime}\right)\right)$ for any $t \leq a_{i}<t^{\prime}$.

In the time intervals $\left(a_{i}, a_{i+1}\right]$ new branches of the fracture can appear; being $S(\cdot)$ constant, they necessarily originate at some point in $S(t)$, for $t \in\left(a_{i}, a_{i+1}\right]$. Together with any new branch, also a new tip appears; by monotonicity of $\Gamma(\cdot)$, for any $t, t^{\prime} \in\left(a_{i}, a_{i+1}\right]$ with $t^{\prime}<t$, it has to be $\operatorname{card}\left(T\left(t^{\prime}\right)\right) \leq \operatorname{card}(T(t))$.

We can again establish a sort of stability from the left: as seen in the discussion in Section 5, for every $t \in\left(a_{i}, a_{i+1}\right]$ there exists $\alpha_{1}(t)<t$ (defined in (5.11)) such that (5.10) holds, i.e.

$$
\operatorname{card}(T(t))=\operatorname{card}\left(T\left(t^{\prime}\right)\right)
$$

for every $t^{\prime} \in\left(\alpha_{1}(t), t\right]$; by (5.10), notice that $\alpha_{1}(t) \geq a_{i}$. Hence we can further subdivide each interval $\left(a_{i}, a_{i+1}\right]$ with a partition $a_{i}=b_{i}^{0}<b_{i}^{1}<\ldots<b_{i}^{n_{i}}=a_{i+1}$ such that

- $\operatorname{card}(T(t))=\operatorname{card}\left(T\left(t^{\prime}\right)\right)$ if $t, t^{\prime} \in\left(b_{i}^{k}, b_{i}^{k+1}\right]$;

- $\operatorname{card}(T(t))<\operatorname{card}\left(T\left(t^{\prime}\right)\right)$ if $a_{i}<t \leq b_{i}^{k}<t^{\prime}<a_{i+1}$.

Actually, above we have proved the following fact.

Lemma 5.7. There exists a partition

$$
0=t_{0}<t_{1}<\ldots<t_{N+1}=1
$$

of the interval $[0,1]$ such that one of the following alternatives holds:

(i) if $t, t^{\prime} \in\left(t_{i}, t_{i+1}\right]$, then $S(t)=S\left(t^{\prime}\right)$ and $\operatorname{card}(T(t))=\operatorname{card}\left(T\left(t^{\prime}\right)\right)$;

(ii) if $t \leq t_{i}<t^{\prime}$, then either $S(t)=S\left(t^{\prime}\right)$ and $\operatorname{card}(T(t))<\operatorname{card}\left(T\left(t^{\prime}\right)\right)$, or $S(t) \neq S\left(t^{\prime}\right)$.

Lemma 5.8. Consider a sequence $\left(\Gamma_{k}\right)_{k} \subset \mathcal{S}$ such that $\Gamma_{k} \stackrel{\mathcal{H}}{\longrightarrow} \widehat{\Gamma}$. Then for every $\psi \in C_{b}(\Omega)$

$$
\int_{\Gamma_{k}} \psi d \mathcal{H}^{1} \rightarrow \int_{\widehat{\Gamma}} \psi d \mathcal{H}^{1}
$$

In other words, the sequence of measures $\mu_{k}:=\mathcal{H}^{1}\left\llcorner\Gamma_{k}\right.$ converges to $\widehat{\mu}:=\mathcal{H}^{1}\llcorner\widehat{\Gamma}$ weakly* in $\mathcal{M}_{b}(\Omega)$.

Proof. It is enough to use the regularity of the curves in $\mathcal{R}_{\eta}$, in particular the parametrization provided by Proposition 3.2.(vi).

Let $\mu:[0,1] \rightarrow \mathcal{M}_{b}(\Omega)$ be the map defined as

$$
\mu(t):=\mathcal{H}^{1}\llcorner\Gamma(t) .
$$


Proposition 5.9. The map $\mu:[0,1] \rightarrow \mathcal{M}_{b}(\Omega)$ belongs to the space $A C\left([0,1] ; \mathcal{M}_{b}(\Omega)\right)$.

Proof. By definition of $\mu$, it results that

$$
|\mu(t)|(\Omega)=\mathcal{H}^{1}(\Gamma(t))=\ell(t),
$$

where $\ell$ was introduced in $(5.5)$ and $\ell \in H^{1}(0,1)$ by Proposition 5.1.

Let $\psi \in C_{0}(\Omega)$. Then for every $0 \leq a<b \leq 1$ it is

$$
\begin{aligned}
|\langle\psi, \mu(b)-\mu(a)\rangle| & =\left|\int_{\Omega} \psi d(\mu(b)-\mu(a))\right|=\left|\int_{\Gamma(b) \backslash \Gamma(a)} \psi d \mathcal{H}^{1}\right| \\
& \leq\|\psi\|_{\infty} \mathcal{H}^{1}(\Gamma(b) \backslash \Gamma(a))=\|\psi\|_{\infty}\left(\mathcal{H}^{1}(\Gamma(b))-\mathcal{H}^{1}(\Gamma(a))\right) \\
& =\|\psi\|_{\infty} \int_{a}^{b} \dot{\ell}(\xi) d \xi .
\end{aligned}
$$

Taking the supremum over all $\psi \in C_{0}(\Omega)$ with $\|\psi\|_{\infty} \leq 1$, we obtain

$$
|\mu(b)-\mu(a)|(\Omega) \leq \int_{a}^{b} \dot{\ell}(t) d t .
$$

Since $\ell \in H^{1}(0,1)$, by the absolute continuity of the integral with respect to the integration domain, we obtain the thesis.

In accordance with the results in [13, Appendix], for a.e. $t \in[0,1]$ there exists

$$
\dot{\mu}(t):=w^{*}-\lim _{s \rightarrow t} \frac{\mu(s)-\mu(t)}{s-t},
$$

and $\dot{\mu}(t) \in \mathcal{M}_{b}(\Omega)$. We mean that for a.e. $t \in[0,1]$ there exists a Radon measure $\dot{\mu}(t) \in \mathcal{M}_{b}(\Omega)$ such that

$$
\langle\psi, \dot{\mu}(t)\rangle=\lim _{s \rightarrow t}\left\langle\psi, \frac{\mu(s)-\mu(t)}{s-t}\right\rangle
$$

for every $\psi \in C_{0}(\Omega)$.

We describe the "structure" of these measures, in order to introduce a distributional notion of velocity.

Proposition 5.10. For a.e. $t \in[0,1]$

$$
\operatorname{supp} \dot{\mu}(t) \subset T(t) \text {. }
$$

Proof. Consider $\hat{t} \in[0,1] \backslash\left\{t_{1}, \ldots, t_{N}\right\}$ for which $\dot{\mu}(\hat{t})$ exists, where $t_{1}, \ldots, t_{N}$ are given in Lemma 5.7. Fix $\psi \in C_{0}(\Omega)$ such that $\operatorname{supp} \psi \subset \Omega \backslash T(\hat{t})$. Taken $r_{1}(\hat{t})$ and $\alpha_{1}(\hat{t})$ as in (5.8) and (5.11) respectively, for every $t \in\left(\alpha_{1}(\hat{t}), \hat{t}\right]$ it is

$$
\Gamma(t) \backslash \bigcup_{p \in T(\hat{t})} B_{r_{1}(\hat{t})}(p)=\Gamma(\hat{t}) \backslash \bigcup_{p \in T(\hat{t})} B_{r_{1}(\hat{t})}(p) .
$$

Let $r \in\left(0, r_{1}(\hat{t})\right)$ be such that

$$
\operatorname{supp} \psi \subset \Omega \backslash \bigcup_{p \in T(\hat{t})} \overline{B_{r}(p)} .
$$


By continuity of the set function $\Gamma(\cdot)$ with respect to the Hausdorff converge, if we repeat for $r$ the discussion done for $r_{1}(\hat{t})$ and $\alpha_{1}(\hat{t})$, we obtain that there exists $t_{r}<\hat{t}$ such that

$$
\Gamma(t) \backslash \bigcup_{p \in T(\hat{t})} B_{r}(p)=\Gamma(\hat{t}) \backslash \bigcup_{p \in T(\hat{t})} B_{r}(p)
$$

for every $t \in\left(t_{r}, \hat{t}\right]$. Therefore

$$
\left\langle\psi, \frac{\mu(t)-\mu(\hat{t})}{t-\hat{t}}\right\rangle=\frac{1}{\hat{t}-t} \int_{\Gamma(\hat{t}) \backslash \Gamma(t)} \psi d x=0
$$

for every $t \in\left(t_{r}, \hat{t}\right)$. Taking the limit as $t \rightarrow \hat{t}-$, since $\dot{\mu}(\hat{t})$ exists we get $\langle\psi, \dot{\mu}(\hat{t})\rangle=$ 0 .

We have shown that, for every $t \in(0,1)$ for which $\dot{\mu}(t)$ exists, if $\psi \in C_{0}(\Omega)$ with $\operatorname{supp} \psi \subset \Omega \backslash T(t)$ then $\langle\psi, \dot{\mu}(t)\rangle=0$. Therefore supp $\dot{\mu}(t) \subset T(t)$.

As a consequence of Proposition 5.10, for a.e. $t \in(0,1)$

$$
\dot{\mu}(t) \ll \mathcal{H}^{0}\llcorner T(t) .
$$

Definition 5.11. We call (distributional) velocity of the crack tip $p \in T(t)$ the value $v(t, p)$, where

$$
\dot{\mu}(t)=\sum_{p \in T(t)} v(t, p) \delta_{p}
$$

and $\delta_{x}$ is the Dirac measure concentrated at $x \in \mathbb{R}^{2}$.

Now we pass to the second approach for the description of the front velocity, which will lead to an equivalent definition.

Consider $\hat{t} \in\left(t_{i}, t_{i+1}\right)$, with $t_{i}$ introduced in Lemma 5.7, and $r_{1}(\hat{t}), \alpha_{1}(\hat{t})$ as in (5.8) and (5.11). Fixed $p \in T(\hat{t})$, we can describe the curve

$$
\Gamma(\hat{t}) \cap \overline{B_{r_{1}(\hat{t})}(p)} \in \mathcal{R}_{\eta}
$$

by means of an arc-length parametrization $\gamma:\left[0, L^{\hat{t}, p}\right] \rightarrow \mathbb{R}^{2}$ (here $L^{\hat{t}, p}:=\mathcal{H}^{1}(\Gamma(\hat{t}) \cap$ $\left.\left.\overline{B_{r_{1}(\hat{t})}(p)}\right)\right)$ and an increasing function $\sigma:\left[\alpha_{1}(\hat{t}), \hat{t}\right] \rightarrow\left[0, L^{\hat{t}, p}\right]$ such that for every $t \in\left(\alpha_{1}(\hat{t}), \hat{t}\right]$ it is

$$
\sigma(t)=\mathcal{H}^{1}\left(\Gamma(t) \cap \overline{B_{r_{1}(\hat{t})}(p)}\right) \text { and } \gamma(\sigma(t))=p(t),
$$

where $p(t)$ is the unique element in $T(t) \cap B_{r_{1}(\hat{t})}(p)$. Since the curves in $\mathcal{R}_{\eta}$ belong to $W^{2, \infty}$ and $\ell(\cdot)=\mathcal{H}^{1}(\Gamma(\cdot))$ is in $H^{1}(0,1)$, it results that $\gamma \in W^{2, \infty}$ and $\sigma \in$ $H^{1}\left(\alpha_{1}(\hat{t}), \hat{t}\right)$, hence

$$
\gamma(\sigma(\cdot)) \in H^{1}\left(\left(\alpha_{1}(\hat{t}), \hat{t}\right) ; \mathbb{R}^{2}\right) .
$$

Then, for a.e. $t \in\left(\alpha_{1}(\hat{t}), \hat{t}\right)$, we define the velocity of the crack tip $p(t)$ as

$$
\mathbf{v}(t, p(t)):=\dot{\sigma}(t) \dot{\gamma}(\sigma(t))
$$

and

$$
\tilde{v}(t, p(t)):=|\mathbf{v}(t, p(t))|=\dot{\sigma}(t) .
$$

It is not difficult to see that the two notions (5.20) and (5.21) coincide, i.e.

$$
v(t, p(t))=\tilde{v}(t, p(t))
$$


for a.e. $t \in[0,1]$. Indeed, assume that $\dot{\mu}(t)$ and $\dot{\sigma}(t)$ exist for some $t \in[0,1] \backslash$ $\left\{t_{1}, \ldots, t_{N}\right\}$, with $t_{i}$ as in Lemma 5.7. For $s \in\left(\alpha_{1}(t), t\right)$ it is $\Gamma(t) \backslash \Gamma(s)=$ $\gamma(\sigma((s, t]))$. Fixed $p(t) \in T(t)$, for $\psi \in C_{b}(\Omega)$ with $\operatorname{supp} \psi \subset B_{r_{1}(t)}(p(t))$ and $\psi(p(t))=1$ it is

$$
\begin{aligned}
\frac{1}{t-s} \int_{\Gamma(t) \backslash \Gamma(s)} \psi d \mathcal{H}^{1} & =\frac{1}{t-s} \int_{s}^{t} \psi(\gamma(\sigma(\xi)))\left|\frac{d}{d \xi}(\gamma(\sigma(\xi)))\right| d \xi \\
& =\frac{1}{t-s} \int_{s}^{t} \psi(\gamma(\sigma(\xi))) \dot{\sigma}(\xi) d \xi .
\end{aligned}
$$

As $s \nearrow t$, the left-hand side converges to

$$
\langle\psi, \dot{\mu}(t)\rangle=\left\langle\psi, \sum_{p \in T(t)} v(t, p) \delta_{p}\right\rangle=\psi(p(t)) v(t, p(t))=v(t, p(t)),
$$

while the right-hand side to

$$
\psi(\gamma(\sigma(t))) \dot{\sigma}(t)=\psi(p(t)) \tilde{v}(t, p(t))=\tilde{v}(t, p(t)) .
$$

Hence (5.22) is proved.

Similarly to the map $\mu:[0,1] \rightarrow \mathcal{M}_{b}(\Omega)$ defined in (5.19), we introduce $\mu_{\tau}$ : $[0,1] \rightarrow \mathcal{M}_{b}(\Omega)$ as

$$
\mu_{\tau}(t):=\mathcal{H}^{1}\left\llcorner\Gamma_{\tau}(t) .\right.
$$

Lemma 5.8 and (5.1) imply that

$$
\mu_{\tau}(t) \rightarrow \mu(t)
$$

weakly* in $\mathcal{M}_{b}(\Omega)$, for every $t \in[0,1]$. Observe that if $r \in(0, \eta)$, then for every $x \in \Omega$

$$
\mu(t)\left(\partial B_{r}(x)\right)=0 .
$$

Indeed, being $r<\eta$, the constraint on the curvature of the curves $K \in \mathcal{R}_{\eta}$ implies that the set $K \cap \partial B_{r}(x)$ contains finitely many points, and consequently the same holds for the set $\Gamma \cap \partial B_{r}(x)$ for every $\Gamma \in \mathcal{S}$. Then, by (5.24) and (5.25), we obtain that

$$
\mu_{\tau}(t)\left(B_{r}(x)\right) \rightarrow \mu(t)\left(B_{r}(x)\right)
$$

for every $r \in(0, \eta)$ and $x \in \Omega$.

Lemma 5.12. Let $\hat{t} \in \mathcal{A}, r(\hat{t})$ given by Lemma 5.6 and $p \in M T(\hat{t})$. For every $t \in(\alpha(\hat{t}), \hat{t})$ and $\tau$ such that $t+\tau \in(\alpha(\hat{t}), \hat{t}]$, the set

$$
\left(\Gamma_{\tau}(t+\tau) \backslash \Gamma_{\tau}(t)\right) \cap B_{r(\hat{t})}(p)
$$

is either empty or connected.

Proof. Assume that the set is not empty. By choice of $\hat{t}$, it is $\Gamma_{\tau}(\hat{t}) \cap \overline{B_{r(\hat{t})}(p)} \in \mathcal{R}_{\eta}$ and $T_{\tau}(\hat{t}) \cap B_{r(\hat{t})}(p)=\left\{p_{\tau}(\hat{t})\right\}$. If for some $t$ and $\tau$ the set in (5.26) has two or more connected components, then they must be separated by points or arcs of curve contained in $\Gamma_{\tau}(t)$ : there exists $\mathfrak{c}$ connected component of $\Gamma_{\tau}(t)$ with $\mathfrak{c}$ strictly contained in $B_{r(\hat{t})}(p)$ and $\overline{\mathfrak{c}} \in \mathcal{R}_{\eta}$ (because $\overline{\mathfrak{c}} \subset \Gamma_{\tau}(\hat{t}) \cap \overline{B_{r(\hat{t})}(p)} \in \mathcal{R}_{\eta}$ ). By the fact that (5.13) is verified for our choice of $r(\hat{t})$, it is $\mathcal{H}^{1}(\mathfrak{c})<\lambda$. However this is impossible, since $\Gamma_{\tau}(t) \in \mathcal{S}$ and all its connected components must have length at least $\lambda$. 
Hence, by the previous lemma we conclude that, for $\tau$ sufficiently small and $t \in(\alpha(\hat{t}), \hat{t}]$, it is

$$
\left(\Gamma_{\tau}(t+\tau) \backslash \Gamma_{\tau}(t)\right) \cap B_{r(\hat{t})}(p)=\mathfrak{c}_{\tau}^{p_{\tau}(t)}
$$

for the connected component $\mathfrak{c}_{\tau}^{p_{\tau}(t)} \in \mathcal{C}\left(\Gamma_{\tau}(t), \Gamma_{\tau}(t+\tau)\right)$ with $p_{\tau}(t) \in \mathfrak{c}_{\tau}^{p_{\tau}(t)}$.

We introduce the following notion of discrete velocity. For any $p \in T_{\Gamma_{\tau}(t+\tau)} \backslash T_{\Gamma_{\tau}(t)}$ set

$$
v_{\tau}(t, p):=\frac{1}{\tau} \mathcal{H}^{1}\left(\mathfrak{c}_{\tau}^{p}\right)
$$

where, as above, $\mathfrak{c}_{\tau}^{p}$ is the connected component in $\mathcal{C}\left(\Gamma_{\tau}(t), \Gamma_{\tau}(t+\tau)\right)$ containing $p$. If $p \in T_{\Gamma_{\tau}(t+\tau)} \cap T_{\Gamma_{\tau}(t)}$, simply set $v_{\tau}(t, p):=0$.

Remark 5.13. Let us underline once more that the connected components $\mathfrak{c}_{\tau}^{p}$ above might not be $C^{1,1}$ arcs of curve, but they might kink or contain several branches.

In conclusion of the section, we establish a result which relates $v_{\tau}$ and $v$ in small time intervals.

Proposition 5.14. For every $\hat{t} \in[0,1] \backslash\left(\mathcal{A}_{0} \backslash \mathcal{A}\right)$ let $\alpha(\hat{t})$ be as in Lemmas 5.5 or 5.6. Then for every interval $(a, b) \subset(\alpha(\hat{t}), \hat{t})$ it holds

$$
\int_{a}^{b} v(t, p(t))^{2} d t \leq \liminf _{\tau \rightarrow 0} \int_{a}^{b} v_{\tau}\left(t, p_{\tau}(t)\right)^{2} d t
$$

where, if $t \in \mathcal{A}$, then $p(t)$ and $p_{\tau}(t)$ are as in Lemma 5.6.

Proof. Fixed $\hat{t} \in[0,1] \backslash\left(\mathcal{A}_{0} \backslash \mathcal{A}\right)$, consider $p \in T(\hat{t}) \backslash M T(\hat{t})$ (if this set is not empty). Let $\alpha(\hat{t})$ be as Lemma 5.5 or Lemma 5.6 :

$$
\Gamma(t) \cap \overline{B_{r_{1}(\hat{t})}(p)}=\Gamma(\hat{t}) \cap \overline{B_{r_{1}(\hat{t})}(p)}
$$

for all $t \in(\alpha(\hat{t}), \hat{t}]$. Using the definition (5.21) of $\tilde{v}$ and the notation introduced therein, it results that $\tilde{v}(t, p(t))=\tilde{v}(t, p)=0$. Since (5.22) holds true, it is

$$
v(t, p(t))=\tilde{v}(t, p(t))=0
$$

for a.e. $t \in(\alpha(\hat{t}), \hat{t}]$. Therefore for any $(a, b) \subset(\alpha(\hat{t}), \hat{t})$ it is

$$
0=\int_{a}^{b} v(t, p(t))^{2} d t \leq \int_{a}^{b} v_{\tau}\left(t, p_{\tau}(t)\right)^{2} d t
$$

If $\hat{t} \in \mathcal{A}$ and $p \in M T(\hat{t})$, let $\alpha(\hat{t}), \nu(\hat{t}), r(\hat{t}), p(t), p_{\tau}(t)$ be as in Lemma 5.6. For $t \in(\alpha(\hat{t}), \hat{t})$, by Lemma 5.6 and Lemma 5.12 it results that it is either $v_{\tau}\left(t, p_{\tau}(t)\right)=0$ or $v_{\tau}\left(t, p_{\tau}(t)\right)=\frac{1}{\tau} \mathcal{H}^{1}\left(\mathfrak{c}_{\tau}^{p_{\tau}(t)}\right)$, where $\mathfrak{c}_{\tau}^{p_{\tau}(t)}$ is the connected component in $\mathcal{C}\left(\Gamma_{\tau}(t), \Gamma_{\tau}(t+\right.$ $\tau)$ ) containing $p_{\tau}(t)$. Set

$$
\begin{aligned}
& \tilde{\ell}_{\tau}^{p, r(\hat{t})}(t):=\mathcal{H}^{1}\left(\Gamma_{\tau}(t) \cap B_{r(\hat{t})}(p)\right)+\frac{t-i \tau}{\tau} \mathcal{H}^{1}\left(\left(\Gamma_{\tau}(t+\tau) \backslash \Gamma_{\tau}(t)\right) \cap B_{r(\hat{t})}(p)\right) \\
& \ell^{p, r(\hat{t})}(t):=\mathcal{H}^{1}\left(\Gamma(t) \cap B_{r(\hat{t})}(p)\right)=\mu(t)\left(B_{r(\hat{t})}(p)\right) .
\end{aligned}
$$

Arguing as in Proposition 5.1, it results that $\ell^{p, r(\hat{t})} \in H^{1}(0,1)$ and

$$
\tilde{\ell}_{\tau}^{p, r(\hat{t})}(\cdot) \rightarrow \ell^{p, r(\hat{t})}(\cdot)
$$

weakly in $H^{1}(0,1)$. 
For $\tau$ small enough $\mathfrak{c}_{\tau}^{p_{\tau}(t)} \subset B_{r(\hat{t})}(p)$, so that

$$
\dot{\tilde{\ell}}_{\tau}^{p, r(\hat{t})}(t)=\frac{1}{\tau} \mathcal{H}^{1}\left(\mathfrak{c}_{\tau}^{p_{\tau}(t)} \cap B_{r(\hat{t})}(p)\right)=\frac{1}{\tau} \mathcal{H}^{1}\left(\mathfrak{c}_{\tau}^{p_{\tau}(t)}\right)=v_{\tau}\left(t, p_{\tau}(t)\right) .
$$

By definition of $\ell^{p, r(\hat{t})}$, for a.e. $t \in(\alpha(\hat{t}), \hat{t})$ we have

$$
\dot{\ell}^{p, r(\hat{t})}(t)=\dot{\mu}(t)\left(B_{r(\hat{t})} p\right)=\sum_{q \in T(t)} v(t, q) \delta_{q}\left(B_{r(\hat{t})}(p)\right)=v(t, p(t)),
$$

where the last equality is due to the fact that $T(t) \cap B_{r(\hat{t})}(p)=\{p(t)\}$ for $t \in(\alpha(t), t)$ (see Lemma 5.6). By (5.29), in particular it is

$$
v_{\tau}\left(\cdot, p_{\tau}(\cdot)\right) \rightarrow v(\cdot, p(\cdot))
$$

weakly in $L^{2}(\alpha(\hat{t}), \hat{t})$. Hence, by $(5.29)$ for every $(a, b) \subset(\alpha(\hat{t}), \hat{t})$ it is

$$
\begin{aligned}
\int_{a}^{b} v(t, p(t))^{2} d t=\int_{a}^{b}\left|\dot{\ell}^{p, r}(t)\right|^{2} d t & \leq \liminf _{\tau \rightarrow 0} \int_{a}^{b}\left|\dot{\tilde{\ell}}_{\tau}^{p, r}(t)\right|^{2} d t \\
& =\liminf _{\tau \rightarrow 0} \int_{a}^{b} v_{\tau}\left(t, p_{\tau}(t)\right)^{2} d t
\end{aligned}
$$

\section{Properties of the Continuous-time evolution}

In this section we give a characterization of the evolution $t \mapsto(u(t), \Gamma(t))$ selected in Section 5. Indeed, the approximation by means of the discrete-time evolutions obtained in Section 4 provides $(u(t), \Gamma(t))$ with further interesting properties.

In the following, $\Gamma(t)$ is the family of sets obtained in (5.1), and $u(t)$ is the solution to the problem (5.2).

6.1. Energy inequality. We want to obtain an energy inequality for the continuoustime evolution (see Proposition 6.4). The presence of several branches of the fracture requires a careful control of the approximation process by the discrete-time evolutions, in order to obtain the proper dissipation energy due to the crack increase rate.

Rewritten with the notations introduced in Section 5, the inequality (4.11) has the form

$$
\begin{gathered}
\left\|\nabla u_{\tau}(b)\right\|^{2}+\mathcal{H}^{1}\left(\Gamma_{\tau}(b)\right)+\int_{i \tau}^{k \tau} \sum_{p \in T_{\tau}(t)} v_{\tau}(t, p)^{2} d t \\
\leq\left\|\nabla u_{\tau}(a)\right\|^{2}+\mathcal{H}^{1}\left(\Gamma_{\tau}(a)\right)+2 \int_{i \tau}^{k \tau}\left\langle\nabla u_{\tau}(t), \nabla \dot{w}(t)\right\rangle d t+\varpi(\tau),
\end{gathered}
$$

where $a<b, 0 \leq i \tau \leq a<(i+1) \tau$ and $k \tau \leq b<(k+1) \tau \leq T$ for some $i, k \in\left\{0, \ldots, N_{\tau}\right\}, i \leq k$.

Lemma 6.1. For every $t \in[0,1)$ it is $\Gamma_{\tau}(t+\tau) \stackrel{\mathcal{H}}{\longrightarrow} \Gamma(t)$ as $\tau \rightarrow 0$.

Proof. Fix $t \in[0,1)$ and let $i \in\left\{0, \ldots, N_{\tau}\right\}$ be such that $i \tau \leq t<(i+1) \tau$. Set

$$
\widetilde{\Gamma}(t):=\mathcal{H}-\lim _{\tau \rightarrow 0} \Gamma_{\tau}(t+\tau),
$$


which exists (up to subsequences) and belongs to the family $\mathcal{S}$ by compactness of this class (see Theorem 3.8).

By contradiction, assume $\widetilde{\Gamma}(t) \backslash \Gamma(t) \neq \varnothing$. Being $\Gamma_{\tau}(t) \subset \Gamma_{\tau}(t+\tau)$, the limit sets verify the same inclusion, i.e. $\Gamma(t) \subset \widetilde{\Gamma}(t)$. By continuity (with respect to the Hausdorff convergence) of the measure $\mathcal{H}^{1}$ restricted to sets in $\mathcal{S}$ (see Lemma 3.14), we have

$$
\begin{aligned}
0 & \leq \mathcal{H}^{1}(\widetilde{\Gamma}(t) \backslash \Gamma(t))=\mathcal{H}^{1}(\widetilde{\Gamma}(t))-\mathcal{H}^{1}(\Gamma(t)) \\
& =\lim _{\tau \rightarrow 0} \mathcal{H}^{1}\left(\Gamma_{\tau}(t+\tau)\right)-\lim _{\tau \rightarrow 0} \mathcal{H}^{1}\left(\Gamma_{\tau}(t)\right)=\lim _{\tau \rightarrow 0} \mathcal{H}^{1}\left(\Gamma_{\tau}(t+\tau) \backslash \Gamma_{\tau}(t)\right) \\
& =\lim _{\tau \rightarrow 0} \mathcal{H}^{1}\left(\Gamma_{\tau}^{i+1} \backslash \Gamma_{\tau}^{i}\right)=\lim _{\tau \rightarrow 0} \sum_{\mathfrak{c} \in \mathcal{C}\left(\Gamma_{\tau}^{i}, \Gamma_{\tau}^{i+1}\right)} \mathcal{H}^{1}(\mathfrak{c})=\lim _{\tau \rightarrow 0} \int_{i \tau}^{(i+1) \tau} \dot{\tilde{\ell}}_{\tau}(\xi) d \xi \\
& \leq \lim _{\tau \rightarrow 0} \tau^{1 / 2}\left(\int_{i \tau}^{(i+1) \tau}\left|\dot{\tilde{\ell}}_{\tau}(\xi)\right|^{2} d \xi\right)^{1 / 2} \leq C \lim _{\tau \rightarrow 0} \tau^{1 / 2}=0,
\end{aligned}
$$

where the last inequality is due to Lemma 4.7.

Hence the set $\widetilde{\Gamma}(t) \backslash \Gamma(t)$ is composed by isolated points, which contradicts the fact that $\widetilde{\Gamma}(t) \in \mathcal{S}$ (Condition 3.6.(iii) is not satisfied). Therefore $\widetilde{\Gamma}(t)=\Gamma(t)$, which, taking into account (6.2), concludes the proof.

Lemma 6.2. For any $t \in[0,1)$ the functions $\nabla u_{\tau}(t)$ and $\nabla u_{\tau}(t+\tau)$ converge to $\nabla u(t)$ strongly in $L^{2}\left(\Omega ; \mathbb{R}^{2}\right)$ as $\tau \rightarrow 0$.

Proof. Fix $t \in[0,1)$ and for every $\tau$ let $i \in\left\{0, \ldots, N_{\tau}\right\}$ be such that $i \tau \leq t<$ $(i+1) \tau$. We already proved in $(5.3)$ that $\nabla u_{\tau}(t) \rightarrow \nabla u(t)$ strongly in $L^{2}\left(\Omega ; \mathbb{R}^{2}\right)$.

Concerning the other claim, we argue as for (5.3): $u(t+\tau)$ is solution to the problem

$$
\begin{cases}\Delta v=0 & \text { in } \Omega \backslash \Gamma_{\tau}^{i+1} \\ v=w((i+1) \tau) & \text { on } \partial_{D} \Omega \\ \frac{\partial v}{\partial \nu}=0 & \text { on } \Gamma_{\tau}^{i+1} .\end{cases}
$$

Then, in order to consider again Lemma 4.3, we notice that $w((i+1) \tau) \rightarrow w(t)$ strongly in $H^{1}(\Omega)$ and, by Lemma $6.1, \Gamma_{\tau}^{i+1}=\Gamma_{\tau}(t+\tau) \stackrel{\mathcal{H}}{\longrightarrow} \Gamma(t)$.

The only remaining term to analyze is the dissipation energy due to the crack growth rate. Then we will have all the tiles to recompose the mosaic. In the following we apply the results at the end of Subsection 5.1.

Let $t_{i}$ be defined as in Lemma 5.16. The set

$$
F:=\left\{t_{0}, \cdots, t_{N}\right\} \cup\left(\mathcal{A}_{0} \backslash \mathcal{A}\right)
$$

is finite (see Lemma 5.3). We write $F=\left\{t_{0}^{\prime}, \ldots, t_{N_{1}}^{\prime}\right\}$ with $t_{i}^{\prime}<t_{i+1}^{\prime}$ and for $t \in\left(t_{i}^{\prime}, t_{i+1}^{\prime}\right)$ we define

$$
I(t)=(\alpha(t), t] \cap\left(t_{i}^{\prime}, t_{i+1}^{\prime}\right),
$$

where $\alpha(t)$ is given by Lemma 5.5 if $t \notin \mathcal{A}_{0}$ and by Lemma 5.6 if $t \in \mathcal{A}$. The following fact holds: 
Lemma 6.3. For every $\tilde{t} \in\left(t_{i}^{\prime}, t_{i+1}^{\prime}\right)$ there exists a countable set $A(\tilde{t}) \subset\left(t_{i}^{\prime}, t_{i+1}^{\prime}\right)$ such that

$$
\left(t_{i}^{\prime}, \tilde{t}\right]=\bigcup_{t \in A(\tilde{t})} I(t)
$$

and $I(t) \cap I\left(t^{\prime}\right)=\varnothing$ for every $t, t^{\prime} \in A(\tilde{t}), t \neq t^{\prime}$.

Proof. Fix $\tilde{t} \in\left(t_{i}^{\prime}, t_{i+1}^{\prime}\right)$ and define

$$
\iota_{\tilde{t}}:=\inf \left\{t \in\left[t_{i}^{\prime}, \tilde{t}\right]:(t, \tilde{t}] \text { can be covered by countably many disjoint } I(\cdot)\right\} \text {. }
$$

Of course $\iota_{\tilde{t}}<\tilde{t}$ since it is enough to consider $I(\tilde{t})$ to obtain that $\iota_{\tilde{t}} \leq \inf I(\tilde{t})$.

By contradiction, assume that $\iota_{\tilde{t}}>t_{i}^{\prime}$. Then the set $I\left(\iota_{\tilde{t}}\right) \cup\left(\iota_{\tilde{t}}, \tilde{t}\right]$ is an interval of the form $(a, \tilde{t}]$, is covered by (at most) countably many disjoint intervals $I(t)$ and

$$
\inf \left(I\left(\iota_{\tilde{t}}\right) \cup\left(\iota_{\tilde{t}}, \tilde{t}\right]\right)=\inf I\left(\iota_{\tilde{t}}\right)<\iota_{\tilde{t}},
$$

in contradiction to the definition (6.3). Therefore $\iota_{\tilde{t}}=t_{i}^{\prime}$.

We want to establish the following lower semicontinuity result about the dissipation at the crack front: for $(a, b) \subset(0,1)$

$$
\int_{a}^{b} \sum_{p \in T(t)} v(t, p)^{2} d t \leq \liminf _{\tau \rightarrow 0} \int_{a}^{b} \sum_{p \in T_{\tau}(t)} v_{\tau}(t, p)^{2} d t
$$

We first prove it in a time interval $(a, b) \subset I(\hat{t})$ for any $\hat{t} \in(0,1) \backslash F$, then we extend it to the case $(a, b) \subset\left(t_{i}^{\prime}, t_{i+1}^{\prime}\right)$ and finally to $(a, b) \subset(0,1)$.

If $\hat{t} \in(0,1) \backslash \mathcal{A}_{0}$, then Proposition 5.14, and in particular (5.28), provides the inequality in $I(\hat{t}) \cap(a, b)$ :

$$
0=\int_{I(\hat{t}) \cap(a, b)} \sum_{p \in T(t)} v(t, p)^{2} d t \leq \int_{I(\hat{t}) \cap(a, b)} \sum_{p \in T_{\tau}(t)} v_{\tau}(t, p)^{2} d t
$$


If $\hat{t} \in \mathcal{A}$, then applying again Proposition 5.14 we obtain:

$$
\begin{aligned}
\int_{I(\hat{t}) \cap(a, b)} \sum_{p \in T(t)} v(t, p)^{2} d t= & \int_{I(\hat{t}) \cap(a, b)} \sum_{p \in M T(t)} v(t, p)^{2} d t \\
& +\int_{I(\hat{t}) \cap(a, b)} \sum_{p \in T(t) \backslash M T(t)} v(t, p)^{2} d t \\
= & \int_{I(\hat{t}) \cap(a, b)} \sum_{p \in M T(\hat{t})} v(t, p(t))^{2} d t \\
& +\int_{I(\hat{t}) \cap(a, b)} \sum_{p \in T(\hat{t}) \backslash M T(\hat{t})} v(t, p(t))^{2} d t \\
= & \sum_{p \in M T(\hat{t})} \int_{I(\hat{t}) \cap(a, b)} v(t, p(t))^{2} d t+0 \\
\leq & \sum_{p \in M T(\hat{t})} \liminf _{\tau \rightarrow 0} \int_{I(\hat{t}) \cap(a, b)} v_{\tau}\left(t, p_{\tau}(t)\right)^{2} d t \\
\leq & \liminf _{\tau \rightarrow 0} \sum_{p \in M T(\hat{t})} \int_{I(\hat{t}) \cap(a, b)} v_{\tau}\left(t, p_{\tau}(t)\right)^{2} d t \\
\leq & \liminf _{\tau \rightarrow 0} \int_{I(\hat{t}) \cap(a, b)} \sum_{p \in T_{\tau}(t)} v_{\tau}(t, p)^{2} d t .
\end{aligned}
$$

Assume now that $(a, b) \subset\left(t_{i}^{\prime}, t_{i+1}^{\prime}\right)$ and consider a sequence $\hat{t}_{k} \nearrow b$. Using the two inequalities above, the countable additivity of the integral and Lemma 6.3, we have

$$
\begin{aligned}
\int_{a}^{\hat{t}_{k}} \sum_{p \in T(t)} v(t, p)^{2} d t & =\sum_{\hat{t} \in A\left(\hat{t}_{k}\right)} \int_{I(\hat{t}) \cap(a, b)} \sum_{p \in T(t)} v(t, p)^{2} d t \\
& \leq \sum_{\hat{t} \in A\left(\hat{t}_{k}\right)}\left(\liminf _{\tau \rightarrow 0} \int_{I(\hat{t}) \cap(a, b)} \sum_{p \in T_{\tau}(t)} v_{\tau}(t, p)^{2} d t\right) \\
& \leq \liminf _{\tau \rightarrow 0}\left(\sum_{\hat{t} \in A\left(\hat{t}_{k}\right)} \int_{I(\hat{t}) \cap(a, b)} \sum_{p \in T_{\tau}(t)} v_{\tau}(t, p)^{2} d t\right) \\
& =\liminf _{\tau \rightarrow 0} \int_{a}^{\hat{t}_{k}} \sum_{p \in T_{\tau}(t)} v_{\tau}(t, p)^{2} d t \\
& \leq \liminf _{\tau \rightarrow 0} \int_{a}^{b} \sum_{p \in T_{\tau}(t)} v_{\tau}(t, p)^{2} d t .
\end{aligned}
$$

As $k \rightarrow+\infty$, we get $(6.4)$ when $(a, b) \subset\left(t_{i}^{\prime}, t_{i+1}^{\prime}\right)$.

Finally, if $(a, b) \subset(0,1)$, then it is enough to argue as above in $(a, b) \cap\left(t_{i}^{\prime}, t_{i+1}^{\prime}\right)$ and then sum over $i$, in order to obtain that (6.4) holds. 
Proposition 6.4. For all $0 \leq a<b \leq 1$, the couple $(u, \Gamma)$ defined by (5.1) and (5.2) satisfies the following energy inequality:

$$
\begin{gathered}
\|\nabla u(b)\|^{2}+\mathcal{H}^{1}(\Gamma(b))+\int_{a}^{b} \sum_{p \in T(t)} v(t, p)^{2} d t \\
\leq\|\nabla u(a)\|^{2}+\mathcal{H}^{1}(\Gamma(a))+2 \int_{a}^{b}\langle\nabla u(t), \nabla \dot{w}(t)\rangle d t .
\end{gathered}
$$

Proof. We choose $i$ and $k$ as in (6.1). In the following series of inequalities, we apply in sequence: Lemma 6.2 and Lemma 6.1, together with Lemma 3.14 and the inequality (6.4); the inequality (6.1) (or, equivalently, (4.11) with $j=k+1$ ); again Lemma 6.2 and Lemma 3.14.

$$
\begin{aligned}
& \|\nabla u(b)\|^{2}+\mathcal{H}^{1}(\Gamma(b))+\int_{a}^{b} \sum_{p \in T(t)} v(t, p)^{2} d t \\
\leq & \liminf _{\tau \rightarrow 0}\left(\left\|\nabla u_{\tau}(b+\tau)\right\|^{2}+\mathcal{H}^{1}\left(\Gamma_{\tau}(b+\tau)\right)+\int_{a}^{b} \sum_{p \in T_{\tau}(t)} v_{\tau}(t, p)^{2} d t\right) \\
\leq & \liminf _{\tau \rightarrow 0}\left(\left\|\nabla u_{\tau}(b+\tau)\right\|^{2}+\mathcal{H}^{1}\left(\Gamma_{\tau}(b+\tau)\right)+\int_{i \tau}^{(k+1) \tau} \sum_{p \in T_{\tau}(t)} v_{\tau}(t, p)^{2} d t\right) \\
\leq & \liminf _{\tau \rightarrow 0}\left(\left\|\nabla u_{\tau}(a)\right\|^{2}+\mathcal{H}^{1}\left(\Gamma_{\tau}(a)\right)+2 \int_{i \tau}^{(k+1) \tau}\left\langle\nabla u_{\tau}(t), \nabla \dot{w}(t)\right\rangle d t+\varpi(\tau)\right) \\
= & \|\nabla u(a)\|^{2}+\mathcal{H}^{1}(\Gamma(a))+2 \int_{a}^{b}\langle\nabla u(t), \nabla \dot{w}(t)\rangle d t .
\end{aligned}
$$

6.2. Energy release rate and Griffith's principle. In order to complete the characterization of the evolution process $(u(t), \Gamma(t))$, we aim at obtaining a description in terms of Griffith's theory. In our framework we are able to achieve this goal as long as the crack set does not change direction abruptly, does not bifurcate and does not stay still (see Theorem 6.8). In those situations it is not even clear what would be the proper choice for predicting the direction in which the fracture is more likely to grow (see $[11,12]$ ).

The key functional is the energy release rate, introduced here below. For any $\Gamma \in \mathcal{S}$ and any function $g \in H^{1}(\Omega)$, we consider the elastic energy related to the body $\Omega \backslash \Gamma$ and the boundary displacement $g$, given by

$$
E(g, \Gamma):=\inf \left\{\|\nabla u\|^{2}: u \in H^{1}(\Omega \backslash \Gamma), u=g \text { on } \partial_{D} \Omega\right\} .
$$

For a tip $p \in T_{\Gamma}$, we say that $\widetilde{\Gamma}$ is an extension of $\Gamma$ at $p$ if $\Gamma \subset \widetilde{\Gamma}, \widetilde{\Gamma} \backslash \Gamma$ is connected and there exists $r>0$ as in the definition of crack tip such that $\widetilde{\Gamma} \backslash \Gamma \subset \subset B_{r}(p)$ and $\widetilde{\Gamma} \cap \overline{B_{r}(p)} \in \mathcal{R}_{\eta}$.

Remark 6.5. Notice that any estension $\widetilde{\Gamma}$ belongs to $\mathcal{S}$, at least when $\mathcal{H}^{1}(\widetilde{\Gamma} \backslash \Gamma)$ is small. 
In order to compute the energy release rate at a fixed $p \in T_{\Gamma}$, fix an extension $\widetilde{\Gamma}^{p}$ of $\Gamma$ at $p$ and consider the family $\left(\widetilde{\Gamma}_{s}^{p}\right)_{s}$ of extensions of $\Gamma$ at $p$ such that

$$
\widetilde{\Gamma}_{s}^{p} \subset \widetilde{\Gamma}^{p} \text { and } \mathcal{H}^{1}\left(\widetilde{\Gamma}_{s}^{p} \backslash \Gamma\right)=s .
$$

According to the results in [28], we define the energy release rate at $p$ as

$$
\mathcal{G}(g, \Gamma, p):=-\lim _{s \rightarrow 0^{+}} \frac{E\left(g, \widetilde{\Gamma}_{s}^{p}\right)-E(g, \Gamma)}{s} .
$$

As proved in [28], this limit exists and is independent of the chosen extension $\widetilde{\Gamma}^{p}$.

Fix $i \in\left\{1, \ldots, N_{\tau}\right\}$ and $p \in T_{\Gamma_{\tau}^{i}}$. Consider a family of extensions $\widetilde{\Gamma}_{s}^{p}$ of $\Gamma_{\tau}^{i}$ at $p$, as above. By the minimality property of $\Gamma_{\tau}^{i}$ and $u_{\tau}^{i}$, we obtain

$$
\begin{gathered}
\left\|\nabla u_{\tau}^{i}\right\|^{2}+\mathcal{H}^{1}\left(\Gamma_{\tau}^{i}\right)+\frac{1}{\tau} \sum_{C \in \mathcal{C}\left(\Gamma_{\tau}^{i-1}, \Gamma_{\tau}^{i}\right)}\left(\mathcal{H}^{1}(\mathfrak{c})\right)^{2} \\
\leq\left\|\nabla u_{\Gamma_{s}^{p}}\right\|^{2}+\mathcal{H}^{1}\left(\widetilde{\Gamma}_{s}^{p}\right)+\frac{1}{\tau} \sum_{\mathfrak{c} \in \mathcal{C}\left(\widetilde{\Gamma}_{\tau}^{i-1}, \widetilde{\Gamma}_{s}^{p}\right)}\left(\mathcal{H}^{1}(\mathfrak{c})\right)^{2},
\end{gathered}
$$

where $u_{\widetilde{\Gamma}_{s}^{p}}$ is the minimizer of the problem (6.5) with $g=w(i \tau)$ and $\Gamma=\widetilde{\Gamma}_{s}^{p}$. Set

$$
\mathfrak{c}_{s}^{p}:=\widetilde{\Gamma}_{s}^{p} \backslash \Gamma_{\tau}^{i} .
$$

If $p \in T_{\Gamma_{\tau}^{i}} \cap T_{\Gamma_{\tau}^{i-1}}$, then $\widetilde{\Gamma}_{s}^{p} \backslash \Gamma_{\tau}^{i-1}=\mathfrak{c}_{s}^{p} \cup\left(\Gamma_{\tau}^{i} \backslash \Gamma_{\tau}^{i-1}\right)$ and

$$
\sum_{\mathfrak{c} \in \mathcal{C}\left(\Gamma_{\tau}^{i-1}, \widetilde{\Gamma}_{s}^{p}\right)}\left(\mathcal{H}^{1}(\mathfrak{c})\right)^{2}=\left(\mathcal{H}^{1}\left(\mathfrak{c}_{s}^{p}\right)\right)^{2}+\sum_{\mathfrak{c} \in \mathcal{C}\left(\Gamma_{\tau}^{i-1}, \Gamma_{\tau}^{i}\right)}\left(\mathcal{H}^{1}(\mathfrak{c})\right)^{2} .
$$

Since $\mathcal{H}^{1}\left(\mathfrak{c}_{s}^{p}\right)=s$, by the above relation and (6.7) we obtain

$$
-\frac{\left\|\nabla u_{\widetilde{\Gamma}_{s}^{p}}\right\|^{2}-\left\|\nabla u_{\tau}^{i}\right\|^{2}}{s} \leq 1+\frac{1}{\tau} s .
$$

Therefore, recalling the definition (6.6) of $\mathcal{G}$, as $s \rightarrow 0^{+}$we get

$$
\mathcal{G}\left(w(i \tau), \Gamma_{\tau}^{i}, p\right) \leq 1 \text {. }
$$

Assume now that

$$
p \in T_{\Gamma_{\tau}^{i}} \backslash T_{\Gamma_{\tau}^{i-1}}
$$

Then $p \in \mathfrak{c}^{p}$ for (only) one $\mathfrak{c}^{p} \in \mathcal{C}\left(\Gamma_{\tau}^{i-1}, \Gamma_{\tau}^{i}\right)$. It results that $\mathfrak{c}^{p} \cup \mathfrak{c}_{s}^{p}$ is connected,

$$
\widetilde{\Gamma}_{s}^{p} \backslash \Gamma_{\tau}^{i-1}=\left(\mathfrak{c}^{p} \cup \mathfrak{c}_{s}^{p}\right) \cup\left(\Gamma_{\tau}^{i} \backslash\left(\Gamma_{\tau}^{i-1} \cup \mathfrak{c}^{p}\right)\right)
$$

and

$$
\sum_{\mathfrak{c} \in \mathcal{C}\left(\Gamma_{\tau}^{i-1}, \widetilde{\Gamma}_{s}^{p}\right)}\left(\mathcal{H}^{1}(\mathfrak{c})\right)^{2}=\left(\mathcal{H}^{1}\left(\mathfrak{c}^{p} \cup \mathfrak{c}_{s}^{p}\right)\right)^{2}+\sum_{\substack{\mathfrak{c} \in \mathcal{C}\left(\Gamma_{\tau}^{i-1}, \Gamma_{\tau}^{i}\right) \\ \mathfrak{c} \neq \mathfrak{c} p}}\left(\mathcal{H}^{1}(\mathfrak{c})\right)^{2} .
$$

It follows that

$$
\begin{aligned}
-\frac{\left\|\nabla u_{\widetilde{\Gamma}_{s}^{p}}\right\|^{2}-\left\|\nabla u_{\tau}^{i}\right\|^{2}}{s} & \leq 1+\frac{1}{\tau} \frac{\left(\mathcal{H}^{1}\left(\mathfrak{c}^{p} \cup \mathfrak{c}_{s}^{p}\right)\right)^{2}-\left(\mathcal{H}^{1}\left(\mathfrak{c}^{p}\right)\right)^{2}}{s} \\
& =1+\frac{1}{\tau} \frac{s^{2}+2 s \mathcal{H}^{1}\left(\mathfrak{c}^{p}\right)}{s}
\end{aligned}
$$




$$
-\frac{\left\|\nabla u_{\widetilde{\Gamma}_{s}^{p}}\right\|^{2}-\left\|\nabla u_{\tau}^{i}\right\|^{2}}{s} \leq 1+\frac{1}{\tau} \frac{\left(\mathcal{H}^{1}\left(\mathfrak{c}^{p} \cup \mathfrak{c}_{s}^{p}\right)\right)^{2}-\left(\mathcal{H}^{1}\left(\mathfrak{c}^{p}\right)\right)^{2}}{s}=1+\frac{1}{\tau} \frac{s^{2}+2 s \mathcal{H}^{1}\left(\mathfrak{c}^{p}\right)}{s}
$$

and, as $s \rightarrow 0^{+}$, we get

$$
\mathcal{G}\left(w(i \tau), \Gamma_{\tau}^{i}, p\right) \leq 1+\frac{2}{\tau} \mathcal{H}^{1}\left(\mathfrak{c}^{p}\right) .
$$

If (6.8) is the case, then also the following sets can be considered in the minimization problem (4.3): $\widehat{\Gamma}_{s}^{p} \in \mathcal{S}$ such that $\Gamma_{\tau}^{i-1} \subset \widehat{\Gamma}_{s}^{p} \subset \Gamma_{\tau}^{i}$, the set $\Gamma_{\tau}^{i} \backslash \widehat{\Gamma}_{s}^{p}$ is connected, $p \in \Gamma_{\tau}^{i} \backslash \widehat{\Gamma}_{s}^{p}$ and $\mathcal{H}^{1}\left(\Gamma_{\tau}^{i} \backslash \widehat{\Gamma}_{s}^{p}\right)=s$. In this case we have that

$$
\widehat{\Gamma}_{s}^{p} \backslash \Gamma_{\tau}^{i-1}=\left(\mathfrak{c}^{p} \cap\left(\widehat{\Gamma}_{s}^{p} \backslash \Gamma_{\tau}^{i-1}\right)\right) \cup\left(\Gamma_{\tau}^{i} \backslash\left(\Gamma_{\tau}^{i-1} \cup \mathfrak{c}^{p}\right)\right)
$$

and

$$
\sum_{\mathfrak{c} \in \mathcal{C}\left(\Gamma_{\tau}^{i-1}, \widehat{\Gamma}_{s}^{p}\right)}\left(\mathcal{H}^{1}(\mathfrak{c})\right)^{2}=\left(\mathcal{H}^{1}\left(\mathfrak{c}^{p} \cap\left(\widehat{\Gamma}_{s}^{p} \backslash \Gamma_{\tau}^{i-1}\right)\right)\right)^{2}+\sum_{\substack{\mathfrak{c} \in \mathcal{C}\left(\Gamma_{\tau}^{i-1}, \Gamma_{\tau}^{i}\right) \\ \mathfrak{c} \neq \mathfrak{c} \mathfrak{c}^{p}}}\left(\mathcal{H}^{1}(\mathfrak{c})\right)^{2}
$$

Inequality (6.7) holds even in this case, with $\widehat{\Gamma}_{s}^{p}$ instead of $\widetilde{\Gamma}_{s}^{p}$, and we obtain

$$
\begin{aligned}
-\left(\left\|\nabla u_{\widehat{\Gamma}_{s}^{p}}\right\|^{2}-\left\|\nabla u_{\tau}^{i}\right\|^{2}\right) \leq & \mathcal{H}^{1}\left(\widehat{\Gamma}_{s}^{p}\right)-\mathcal{H}^{1}\left(\Gamma_{\tau}^{i}\right) \\
& +\frac{1}{\tau}\left(\left(\mathcal{H}^{1}\left(\mathfrak{c}^{p} \cap\left(\widehat{\Gamma}_{s}^{p} \backslash \Gamma_{\tau}^{i-1}\right)\right)\right)^{2}-\left(\mathcal{H}^{1}\left(\mathfrak{c}^{p}\right)\right)^{2}\right) \\
= & -s+\frac{1}{\tau}\left(s^{2}-2 s \mathcal{H}^{1}\left(\mathfrak{c}^{p} \cap\left(\widehat{\Gamma}_{s}^{p} \backslash \Gamma_{\tau}^{i-1}\right)\right)\right) .
\end{aligned}
$$

Dividing by $-s$ and letting $s \rightarrow 0^{+}$, since

$$
\mathcal{H}^{1}\left(\mathfrak{c}^{p} \cap\left(\widehat{\Gamma}_{s}^{p} \backslash \Gamma_{\tau}^{i-1}\right)\right) \rightarrow \mathcal{H}^{1}\left(\mathfrak{c}^{p}\right)
$$

we obtain the reverse inequality

$$
\mathcal{G}\left(w(i \tau), \Gamma_{\tau}^{i}, p\right) \geq 1+\frac{2}{\tau} \mathcal{H}^{1}\left(\mathfrak{c}^{p}\right) .
$$

Using the definition of discrete velocity introduced in (5.27), we can restate the above discussion in terms of a discrete Griffith's principle: for every $t \in(0,1)$ and $p_{\tau}(t) \in T_{\tau}(t)$

$$
\begin{aligned}
& v_{\tau}\left(t, p_{\tau}(t)\right) \geq 0 \\
& \mathcal{G}\left(w_{\tau}(t), \Gamma_{\tau}(t), p_{\tau}(t)\right) \leq 1+v_{\tau}\left(t, p_{\tau}(t)\right) \\
& {\left[-\mathcal{G}\left(w_{\tau}(t), \Gamma_{\tau}(t), p_{\tau}(t)\right)+1+v_{\tau}\left(t, p_{\tau}(t)\right)\right] v_{\tau}\left(t, p_{\tau}(t)\right)=0 .}
\end{aligned}
$$

We now look for a similar stability criterion for the continuous-time evolution. We will see that, in the case of moving tips, this is achievable. On the other hand, when dealing with static tips a number of problematic issues might appear.

In [28, Remark 2.5], the following integral formula for the energy release rate is proved. Let $K \in \mathcal{R}_{\eta}$ and $\gamma$ be its arc-length parametrization. Consider $p \in T_{K}$, $p=\gamma\left(\mathcal{H}^{1}(K)\right)$. Then

$$
\begin{aligned}
\mathcal{G}(g, K, p)= & \int_{\Omega \backslash K}\left[\frac{\left(D_{1} u_{K}\right)^{2}-\left(D_{2} u_{K}\right)^{2}}{2}\left(D_{1} V^{1}-D_{2} V^{2}\right)\right. \\
& \left.+D_{1} u_{K} D_{2} u_{K}\left(D_{2} V^{1}+D_{1} V^{2}\right)\right] d x
\end{aligned}
$$


where $u_{K}$ minimizes $E(g, K), \nabla u_{K}=\left(D_{1} u_{K}, D_{2} u_{K}\right)$, and $V=\left(V_{1}, V_{2}\right)$ is any vector field of class $C^{0,1}$ with compact support in $\Omega$ such that $V(\gamma(s))=\dot{\gamma}(s)$ for $s$ in a neighbourhood of $\mathcal{H}^{1}(K)$ (recall that $p=\gamma\left(\mathcal{H}^{1}(K)\right)$ ). This explicit formula will be useful in the sequel.

For the sake of clarity, in the following lemma we report the proof of [28, Theorem 2.12] with slight modifications. We remind that the set $\mathcal{A}$ was introduced in Lemma 5.3.

Lemma 6.6. Fix $\hat{t} \in \mathcal{A}$ and let $\alpha(\hat{t}), \nu(\hat{t}), r(\hat{t})$ and $p_{\tau}(t)$ be as in Lemma 5.6, and $p(t)$ as in (5.10). Then, for every $p \in M T(\hat{t})$,

$$
\mathcal{G}\left(w_{\tau}(t), \Gamma_{\tau}(t), p_{\tau}(t)\right) \rightarrow \mathcal{G}(w(t), \Gamma(t), p(t))
$$

for every $t \in(\alpha(\hat{t}), \hat{t}]$.

Proof. The strategy is to apply the continuity result about the energy release rate proved in [28]. We set

$$
K_{\tau}^{p}(t):=\Gamma_{\tau}(t) \cap \overline{B_{r(\hat{t})}(p)} \quad \text { and } \quad K^{p}(t):=\Gamma(t) \cap \overline{B_{r(\hat{t})}(p)}
$$

for any $t \in(\alpha(\hat{t}), \hat{t}]$. As seen in Lemma 5.6, $K_{\tau}^{p}(t) \in \mathcal{R}_{\eta}$ and $K_{\tau}^{p}(t) \stackrel{\mathcal{H}}{\longrightarrow} K^{p}(t)$.

Consider $\gamma_{\tau}$ and $\gamma$ arc-length parametrizations of $K_{\tau}^{p}(\hat{t})$ and $K^{p}(\hat{t})$, respectively, with $\gamma_{\tau}(0), \gamma(0) \in \partial B_{r(\hat{t})}(p)$. Set $L:=\mathcal{H}^{1}\left(K^{p}(\hat{t})\right)$, it is $p=\gamma(L)$. Using the notation of Proposition 3.2, $\tilde{\gamma}_{\tau}$ converges to $\gamma$ in the weak ${ }^{*}$ topology of $W^{2, \infty}\left([0, L] ; \mathbb{R}^{2}\right)$. We extend each $K_{\tau}^{p}(\hat{t})$ adding a segment along the tangent direction to the tip $p_{\tau}(\hat{t})=\tilde{\gamma}_{\tau}(L)$ and the same for $K^{p}(\hat{t})$ at $p=\gamma(L)$. Using the Implicit Function Theorem, the bound on the curvature in Definition 3.1.(ii) and the choice of $r(\hat{t})$, these extended curves are graphs of some $C^{1,1}$ scalar functions $\varphi_{\tau}, \varphi$. We fix two coordinate axes such that the extension of $K_{\tau}^{p}(\hat{t})$ is described by $\left(x_{1}, \varphi_{\tau}\left(x_{1}\right)\right)$ and the extension of $K^{p}(\hat{t})$ is described by $\left(x_{1}, \varphi\left(x_{1}\right)\right)$. Fix a cut-off function $\zeta$ supported in $B_{r(\hat{t})}(p)$. Given a point $x=\left(x_{1}, x_{2}\right) \in B_{r(\hat{t})}(p)$, define the vector fields $V_{\tau}(x):=\zeta(x)\left(1, \frac{d}{d x_{1}} \varphi_{\tau}\left(x_{1}\right)\right)$; similarly we define a vector field $V$ related to $\varphi$. By the weak ${ }^{*}$ convergence of $\tilde{\gamma}_{\tau}$ to $\gamma$ in $W^{2, \infty}\left([0, L] ; \mathbb{R}^{2}\right)$, we obtain that $\nabla V_{\tau}$ converges to $\nabla V$ weakly* in $L^{\infty}\left(\Omega ; \mathbb{R}^{4}\right)$.

Observe that, according to the formula (6.12), the vector fields introduced above are suitable for the integral representation of the energy release rate for the curves $K_{\tau}^{p}(t)$ and $K^{p}(t)$ for every $t \in(\alpha(\hat{t}), \hat{t}]$ (and not only for $t=\hat{t}$ ). That is, we have the following equality:

$$
\begin{aligned}
\mathcal{G}\left(w_{\tau}(t), \Gamma_{\tau}(t), p_{\tau}(t)\right)= & \int_{\Omega \backslash \Gamma_{\tau}(t)}\left[\frac{\left(D_{1} u_{\tau}(t)\right)^{2}-\left(D_{2} u_{\tau}(t)\right)^{2}}{2}\left(D_{1} V_{\tau}^{1}-D_{2} V_{\tau}^{2}\right)\right. \\
& \left.+D_{1} u_{\tau}(t) D_{2} u_{\tau}(t)\left(D_{2} V_{\tau}^{1}+D_{1} V_{\tau}^{2}\right)\right] d x
\end{aligned}
$$

and similarly for $\mathcal{G}(w(t), \Gamma(t), p(t))$.

Since $\nabla V_{\tau}$ converges to $\nabla V$ weakly* in $L^{\infty}\left(\Omega ; \mathbb{R}^{4}\right)$ and, as proved in (5.3), $\nabla u_{\tau}(t) \rightarrow \nabla u(t)$ strongly in $L^{2}\left(\Omega ; \mathbb{R}^{2}\right)$ for all $t \in(\alpha(\hat{t}), \hat{t}]$, we obtain the claimed pointwise convergence.

Lemma 6.7. Assume the same hypotheses as in Lemma 6.6. Then, for every $1 \leq$ $q<\infty$

$$
\mathcal{G}(w(\cdot), \Gamma(\cdot), p(\cdot)) \in L^{q}(\alpha(\hat{t}), \hat{t})
$$


and

in $L^{q}(\alpha(\hat{t}), \hat{t})$.

$$
\mathcal{G}\left(w_{\tau}(\cdot), \Gamma_{\tau}(\cdot), p_{\tau}(\cdot)\right) \rightarrow \mathcal{G}(w(\cdot), \Gamma(\cdot), p(\cdot))
$$

Proof. By means of the Dunford-Pettis Theorem (see [3]) and (5.30), we obtain that the functions $v_{\tau}\left(\cdot, p_{\tau}(\cdot)\right)$ are equiintegrable in $(\alpha(\hat{t}), \hat{t})$. Being

$$
0 \leq \mathcal{G}\left(w_{\tau}(t), \Gamma_{\tau}(t), p_{\tau}(t)\right) \leq 1+v_{\tau}\left(t, p_{\tau}(t)\right)
$$

the sequence $\mathcal{G}\left(w_{\tau}(\cdot), \Gamma_{\tau}(\cdot), p_{\tau}(\cdot)\right)$ is equiintegrable too. Then, considering Lemma 6.6, by Vitali's Theorem (see [32, Chapter 6, Exercise 9]) we have that $\mathcal{G}(w(\cdot), \Gamma(\cdot), p(\cdot)) \in$ $L^{1}(\alpha(\hat{t}), \hat{t})$ and

$$
\int_{\alpha(\hat{t})}^{\hat{t}} \mathcal{G}\left(w_{\tau}(t), \Gamma_{\tau}(t), p_{\tau}(t)\right) d t \rightarrow \int_{\alpha(\hat{t})}^{\hat{t}} \mathcal{G}(w(t), \Gamma(t), p(t)) d t
$$

Since $\mathcal{G}$ is non-negative, the last limit means that

$$
\left\|\mathcal{G}\left(w_{\tau}(\cdot), \Gamma_{\tau}(\cdot), p_{\tau}(\cdot)\right)\right\|_{L^{1}(\alpha(\hat{t}), \hat{t})} \rightarrow\|\mathcal{G}(w(\cdot), \Gamma(\cdot), p(\cdot))\|_{L^{1}(\alpha(\hat{t}), \hat{t})} .
$$

Then, applying [3, Proposition 1.33] and the pointwise convergence (6.13), we obtain that

in $L^{1}(\alpha(\hat{t}), \hat{t})$.

$$
\mathcal{G}\left(w_{\tau}(\cdot), \Gamma_{\tau}(\cdot), p_{\tau}(\cdot)\right) \rightarrow \mathcal{G}(w(\cdot), \Gamma(\cdot), p(\cdot))
$$

Finally, observe that, by the integral formula (6.14) for the energy release rate, it results

$$
\mathcal{G}\left(w_{\tau}(\cdot), \Gamma_{\tau}(\cdot), p_{\tau}(\cdot)\right), \mathcal{G}(w(\cdot), \Gamma(\cdot), p(\cdot)) \in L^{\infty}(\alpha(\hat{t}), \hat{t}) .
$$

Indeed, the maps $\nabla V_{\tau}$ and $\nabla V$ are uniformly bounded in $L^{\infty}$ by the $W^{2, \infty}$ norms of $\gamma_{\tau}$ and $\gamma$ introduced in Lemma 6.6; for $\nabla u_{\tau}(t)$ and $\nabla u(t)$ we use (4.12) and (5.4) to have a uniform bound.

The $L^{\infty}$ bound uniform in $\tau$ and the $L^{1}$ convergence proved above are sufficient to conclude.

The concluding main result of this section is proved in the following theorem.

Theorem 6.8. Fix $\hat{t} \in \mathcal{A}$ and let $\alpha(\hat{t}), \nu(\hat{t}), r(\hat{t})$ be as in Lemma 5.6, and $p(t)$ as in (5.10). Then, for every $p \in M T(\hat{t})$, the following conditions hold for a.e. $t \in(\alpha(\hat{t}), \hat{t}):$

$$
\begin{aligned}
& v(t, p(t)) \geq 0 \\
& \mathcal{G}(w(t), \Gamma(t), p(t)) \leq 1+v(t, p(t)) \\
& {[-\mathcal{G}(w(t), \Gamma(t), p(t))+1+v(t, p(t))] v(t, p(t))=0 .}
\end{aligned}
$$

Proof. Fix $t$ such that $\dot{\mu}(t)$ exists. Consider $\psi \in C_{b}(\Omega)$ with $\operatorname{supp} \psi \subset B_{r_{1}(t)}(p(t))$, $\psi \geq 0$ and $\psi(p(t))=1$. Then, by (5.23), it is

$$
v(t, p(t))=\langle\psi, \dot{\mu}(t)\rangle=\lim _{s \nearrow t} \frac{1}{t-s} \int_{\Gamma(t) \backslash \Gamma(s)} \psi d \mathcal{H}^{1} \geq 0,
$$

hence (6.15) holds.

Let $(a, b) \subset(\alpha(\hat{t}), \hat{t})$. By the weak convergence (5.30), Lemma 6.7 and (6.10), we obtain

$$
\int_{a}^{b} \mathcal{G}(w(t), \Gamma(t), p(t)) d t \leq \int_{a}^{b}[1+v(t, p(t))] d t .
$$


If $t^{\prime} \in(\alpha(\hat{t}), \hat{t})$ is a Lebesgue point of the function $-\mathcal{G}(w(\cdot), \Gamma(\cdot), p(\cdot))+1+v(\cdot, p(\cdot))$, by the inequality above we obtain

$$
\begin{aligned}
0 & \leq \lim _{\varepsilon \rightarrow 0+} \frac{1}{\varepsilon} \int_{t^{\prime}-\varepsilon}^{t^{\prime}}[-\mathcal{G}(w(t), \Gamma(t), p(t))+1+v(t, p(t))] d t \\
& =-\mathcal{G}\left(w\left(t^{\prime}\right), \Gamma\left(t^{\prime}\right), p\left(t^{\prime}\right)\right)+1+v\left(t^{\prime}, p\left(t^{\prime}\right)\right) .
\end{aligned}
$$

Therefore (6.16) holds true a.e. in $(\alpha(\hat{t}), \hat{t})$.

The inequalities (6.15) and (6.16) trivially imply

$$
[-\mathcal{G}(w(t), \Gamma(t), p(t))+1+v(t, p(t))] v(t, p(t)) \geq 0 .
$$

Then, considering (6.11), the weak convergence (5.30) and Lemma 6.7, we have the following chain of inequalities

$$
\begin{aligned}
0 \leq & \int_{\alpha(\hat{t})}^{\hat{t}}[-\mathcal{G}(w(t), \Gamma(t), p(t))+1+v(t, p(t))] v(t, p(t)) d t \\
\leq & \lim _{\tau \rightarrow 0} \int_{\alpha(\hat{t})}^{\hat{t}}\left[-\mathcal{G}\left(w_{\tau}(\cdot), \Gamma_{\tau}(\cdot), p_{\tau}(\cdot)\right) v_{\tau}\left(t, p_{\tau}(t)\right)+v_{\tau}\left(t, p_{\tau}(t)\right)\right] d t \\
& +\liminf _{\tau \rightarrow 0} \int_{\alpha(\hat{t})}^{\hat{t}} v_{\tau}\left(t, p_{\tau}(t)\right)^{2} d t \\
\leq & \liminf _{\tau \rightarrow 0} \int_{\alpha(\hat{t})}^{\hat{t}}\left[-\mathcal{G}\left(w_{\tau}(t), \Gamma_{\tau}(t), p_{\tau}(t)\right)+1+v_{\tau}\left(t, p_{\tau}(t)\right)\right] v_{\tau}\left(t, p_{\tau}(t)\right)=0,
\end{aligned}
$$

i.e.

$$
\int_{\alpha(\hat{t})}^{\hat{t}}[-\mathcal{G}(w(t), \Gamma(t), p(t))+1+v(t, p(t))] v(t, p(t)) d t=0 .
$$

Together with (6.18), this equality implies (6.17) for a.e. $t \in(\alpha(\hat{t}), \hat{t})$.

In conclusion, we would like to explain some of the difficulties that arise in the characterization of the behaviour of points in $T(\hat{t}) \backslash M T(\hat{t})$. In general, our method does not provide information about unilateral minimality properties for the continuous time evolution, therefore any property concerning it needs to be derived by the limit behaviour of the discrete-time evolutions.

In case of static tips, we are not able to prove a result as Lemma 5.6, which plays a key role in the proof of the subsequent results. For example, a static tip might be approximated by a discrete-time sequence of cracks that kink near the tip. The approximation procedure suggests that, in this situation, many direction of growth for the crack tip (of the continuous-time evolution) are possible: which would be the preferred one? How to deal with the energy release rate $\mathcal{G}$, which, as proved by Negri [30], depends on the kinking angle?

Unfortunately, in the mathematical setting we proposed it is not possible to avoid this kind of situations and the complete description of the growth process remains an open problem. Anyway, it is not a simple task to introduce further restrictions on the geometrical properties of the crack sets in the class $\mathcal{S}$, without finding some geometrical setting already discussed in the literature (see for example [27, 25]). On the other hand, our geometrical constraints are necessary in order to avoid some mathematical "pathologies" that would arise if branching is admissible and those 
constraints were absent. To our knowledge, this is a first attempt to consider kinking and branching in the framework of Griffith's theory, and much work needs to be done.

Acknowledgements: The author thanks Professor Gianni Dal Maso for many helpful and interesting discussions. This work has been supported by the ERC Advanced Grant n. 290888 QuaDynEvoPro.

\section{REFERENCES}

[1] R.A. Adams, Sobolev spaces. Pure and Applied Mathematics, Vol. 65. Academic Press. New York-London, 1975.

[2] L. Ambrosio, A compactness theorem for a new class of functions of bounded variation. Boll. Un. Mat. Ital. B (7) 3 (1989), no. 4, 857-881.

[3] L. Ambrosio, N. Fusco, D. Pallara, Functions of bounded variation and free discontinuity problems. Oxford Mathematical Monographs. The Clarendon Press, Oxford University Press, New York, 2000.

[4] L. Ambrosio, N. Gigli, G. Savaré, Gradient Flows in Metric Spaces and in the Space of Probability Measures. Second edition. Lectures in Mathematics ETH Zürich. Birkhäuser-Verlag, Basel, 2008.

[5] L. Ambrosio, P. Tilli, Topics on analysis in metric spaces. Oxford Lecture Series in Mathematics and its Applications, 25. Oxford University Press, Oxford, 2004.

[6] D. Bucur, A. Henrot, J. Sokolowski, A. Zochowski, Continuity of the elasticity system solutions with respect to the geometrical domain variations. Adv. Math. Sci. Appl. 11 (2001), no. $1,57-73$.

[7] D. Bucur, N. Varchon, A duality approach for the boundary variation of Neumann problems. SIAM J. Math. Anal. 34 (2002), 460-477.

[8] D. Bucur, N. Varchon, Boundary variation for the Neumann problem. Ann. Scuola Norm. Sup. Pisa Cl. Sci. 29 (2000), 807-822.

[9] D. Bucur, N. Varchon, Stabilité de la solution d'un problème de Neumann pour des variations de frontière. C. R. Acad. Sci. Paris Sér. I Math. 331 (2000), no. 5, 371-374.

[10] Chambolle A.: A Density Result in Two-Dimensional Linearized Elasticity, and Applications. Arch. Ration. Mech. Anal. 167 (2003), 211-233.

[11] A. Chambolle, G. A. Francfort, J.-J. Marigo, Revisiting energy release rates in brittle fracture. J. Nonlinear Sci. 20 (2010), no. 4, 395-424.

[12] A. Chambolle, G. A. Francfort, J.-J. Marigo, When and how do cracks propagate? J. Mech. Phys. Solids 57 (2009), no. 9, 1614-1622.

[13] G. Dal Maso, A. DeSimone, M. G. Mora, Quasistatic evolution problems for linearly elasticperfectly plastic materials, Arch. Ration. Mech. Anal. 180 (2006), no. 2, 237-291.

[14] G. Dal Maso, F. Ebobisse, M. Ponsiglione, A stability result for nonlinear Neumann problems under boundary variations. J. Math. Pures Appl. 982 (2003), no. 5, 503-532.

[15] G. Dal Maso, G. A. Francfort, R. Toader, Quasistatic crack growth in nonlinear elasticity. Arch. Ration. Mech. Anal. 176 (2005), no. 2, 165-225.

[16] G. Dal Maso, G. Lazzaroni, Quasistatic crack growth in finite elasticity with noninterpenetration. Ann. Inst. H. Poincar Anal. Non Linaire 27 (2010), no. 1, 257-290.

[17] G. Dal Maso, R. Toader, A model for the quasi-static growth of brittle fractures: existence and approximation results. Arch. Ration. Mech. Anal. 162 (2002), no. 2, 101-135.

[18] G. Dal Maso, R. Toader, A model for the quasi-static growth of brittle fractures based on local minimization. Math. Models Methods Appl. Sci. 12 (2002), 1773-1800.

[19] K. Falconer, Fractal geometry. Mathematical foundations and applications. Second edition. John Wiley \& Sons, Inc., Hoboken, NJ, 2003.

[20] G. A. Francfort, C. Larsen, Existence and convergence for quasi-static evolution in brittle fracture. Comm. Pure Appl. Math. 56 (2003), no. 10, 1465-1500. 
[21] G. A. Francfort, J. J. Marigo, Revisiting brittle fractures as an energy minimization problem. J. Mech. Phys. Solids 46 (1998), no. 8, 1319-1342.

[22] A. A. Griffith, The phenomena of rupture and flow in solids. Phil. Trans. Roy. Soc. London 18 (1920), 16-98.

[23] D. Knees, A. Mielke, Energy release rate for cracks in finite-strain elasticity. Math. Meth. Appl. Sci. 31 (2008), 501-528.

[24] D. Knees, C. Zanini, A. Mielke, Crack growth in polyconvex materials. Phys. D 239 (2010), no. $15,1470-1484$.

[25] D. Knees, A. Mielke, C. Zanini, On the inviscid limit of a model for crack propagation. Math. Models Methods Appl. Sci. 18 (2008), no. 9, 1529-1569.

[26] C. Larsen, M. Ortiz, C. L. Richardson, Fracture paths from front kinetics: relaxation and rate independence. Arch. Ration. Mech. Anal. 193 (2009), no. 3, 539-583.

[27] G. Lazzaroni, R. Toader, A model for crack propagation based on viscous approximations. Math. Models Methods Appl. Sci., 21 (2011), no. 10, 2019-2047.

[28] G. Lazzaroni, R. Toader, Energy release rate and stress intensity factor in antiplane elasticity. Journal de Mathématiques Pures et Appliquées 95 (2011), no. 6, 565-584.

[29] M. Negri, A comparative analysis on variational models for quasi-static brittle crack propagation. Adv. Calc. Var. 3 (2010), 149-212.

[30] M. Negri, Energy release rate along a kinked path. Math. Methods Appl. Sci. 34 (2011), no. 4, 384-396.

[31] M. Negri, C. Ortner, Quasi-static crack propagation by Griffith's criterion. Math. Models Methods Appl. Sci. 18 (2008), 1895-1925.

[32] W. Rudin, Real and complex analysis. Third edition. McGraw-Hill Book Co., New York, 1987.

[33] V. Šverak, On optimal shape design. J. Math. Pures Appl. 72 (1993), 537-551.

[34] R. Toader, C. Zanini, An artificial viscosity approach to quasistatic crack growth. Boll. Unione Mat. Ital. 9 (2009), no. 1, 1-35. 